\title{
A Minority pulls the sample mean: on the individual prevalence of robust group-level cognitive phenomena - the instance of the SNARC effect
}

Krzysztof Cipora $^{1 *}$, Jean-Philippe van Dijck ${ }^{2,3}$, Carrie Georges ${ }^{4}$, Nicolas Masson ${ }^{4,5}$, Silke M. Göbel $^{6,7}$, Klaus Willmes $^{8}$, Mauro Pesenti ${ }^{5}$, Christine Schiltz ${ }^{4}$, \& Hans-Christoph Nuerk ${ }^{9,10}$

${ }^{1}$ Centre for Mathematical Cognition, Loughborough University, Loughborough, UK

2 Department of Experimental Psychology, Ghent University, Belgium

${ }^{3}$ Department of Applied Psychology: Thomas More University of Applied Sciences, Belgium

${ }^{4}$ Institute of Cognitive Science and Assessment, Department of Behavioral and Cognitive Science, University of Luxembourg, Luxembourg

${ }^{5}$ Institut de Recherche en Sciences Psychologiques and Institute of Neuroscience, Université catholique de Louvain, Louvain-la-Neuve, Belgium

${ }^{6}$ Department of Psychology, University of York, York, UK

${ }^{7}$ Department of Special Needs Education, University of Oslo, Norway

${ }^{8}$ Department of Neurology, University Clinic, RWTH Aachen University, Aachen Germany

${ }^{9}$ Department of Psychology, University of Tuebingen, Tuebingen, Germany

${ }^{10}$ LEAD Graduate School \& Research Network, University of Tuebingen, Tuebingen, Germany

${ }^{*}$ Corresponding author: Centre for Mathematical Cognition, Wavy top Building. LE11 3TU United Kingdom. E-mail: k.cipora@lboro.ac.uk 


\begin{abstract}
The aim of cognitive psychology is to obtain insights into human cognition in general. For this purpose, group-studies are typically conducted on representative samples, such that the results can be generalized to the population. Within this approach, individual differences are typically neglected or considered to be error variance. Little is known about the prevalence of group-level phenomena at the individual level. Such information is nevertheless important for claims about the universality of phenomena, as, in theory, significant effects at the group-level can be driven by a minority of participants. Here we used a uniform analysis of 18 existing data sets revealing a wellreplicated phenomenon in numerical cognition at the group level, the so-called SNARC (Spatial-Numerical Association of Response Codes) effect, to investigate its prevalence at the individual level. Three methods of analyzing the presence of this effect at the individual level were utilized: one psychometric and two bootstrapping methods. The results suggest that the group-level SNARC effect is driven by a minority of individuals $(\leq 45 \%)$. Our findings are pertinent to an important theoretical question: whether group-level effects are reflecting general principles of cognition. We discuss advantages and drawbacks of the present methods and their utility in investigating the prevalence of other cognitive phenomena. We posit that testing the presence of robust group-level cognitive effects at the individual level, as well as ensuring their reliable measurement, is an important step towards integrating two traditionally separate experimental and correlational approaches of scientific psychology, as previously proposed by Cronbach in the 1950s.
\end{abstract}

250 words (Limit 250) 
Keywords: individual differences in cognitive phenomena; meta-analytic techniques; confidence intervals for cognitive phenomena; prevalence of cognitive phenomena; bootstrap confidence intervals for cognitive phenomena; reliability of cognitive phenomena; SNARC effect 


\section{INTRODUCTION}

Scientific psychology: experimental vs. correlational approach

In his presidential address to the American Psychological Association, Cronbach (1957) distinguished two disciplines of scientific psychology: experimental and correlational. This important distinction shaped both experimental and differential psychology in the following decades, in particular, the research approaches and questions being asked. The aim of experimental psychology is to identify and understand processes and mental representations that underlie human behavior and reasoning.

The methods of experimental psychology are fundamental to cognitive psychology, as much cognitive research is employing group studies. This so-called "experimental approach" aims to precisely characterize cognitive mechanisms based on the typical or average response to a manipulation of environmental variables. In case statistically robust effects are found, and especially when these effects are replicated multiple times in random and representative samples, conclusions are drawn for the general population.

Besides showing many systematic and robust patterns, human behavior is also characterized by many inter-individual differences. In experimental psychology, these inconsistencies are treated as noise or measurement error when averaged out in the search for the mean group effect. However, by collapsing data across participants, the experimental approach basically ignores, among others, the use of different cognitive strategies for the same task, differences in learning or subjective judgment, as well as the inherent normal variance in ability and capacity. For this reason, using the experimental approach can lead to so-called ecological fallacy, such that one assumes that effects present at a group level manifest themselves at the individual participant level (Fisher et al., 2018). Consequently, a wealth of scientifically and clinically relevant 
information is lost in experimental research and is typically not considered in the psychological theories that are built upon these empirical group-level observations. As pointed out recently by Rouder and Haaf (2020), group-level effects should instead be a starting point, while searching for individual differences should be an even more exciting endeavor for psychologists. To fully characterize the cognitive processes underlying human behavior, one therefore needs to treat between-subject variance as data rather than noise (Sauce \& Matzel, 2013; Thompson-Schill et al., 2005; Vogel \& Awh, 2008). Focusing on individual rather than group data allows researchers not only to characterize the actual prevalence of cognitive effects within the population, but also to relate cognitive abilities to brain structures, chemistry, and functions. This so-called "correlational approach" is therefore increasingly employed in the domain of cognitive psychology (see Goodhew \& Edwards, 2019 for a recent discussion). In other words, while the gap between experimental and differential psychology still exists (see Green et al., 2016 for analysis of recent journal articles), new insights are proposed and new tools are being developed to bridge it.

\section{Individual differences research and the problem of low reliability}

However, quantifying cognitive effects at the individual level to subsequently assess their prevalence in a population is not straightforward. In psychometric terms, an individual's score on a task used to capture a certain cognitive effect is just an estimate of the true effect combined with a measurement error - at least, this is how scores are treated in traditional analyses (see e.g., Mead, 2005). To have an idea about the measurement error in a specific sample, the reliability of the instrument/ test is typically calculated. These estimates can then be used to determine confidence intervals $(\mathrm{Cl})$ around the observed scores, which can allow one to infer which participant exhibits the effect or not (see below for a more detailed description). 
In differential psychology, challenge of reliability is widely recognized: The reliability of an instrument plays an important role in correlational studies. The upper bound of a correlation that can be obtained between two measures is set by their reliabilities (Mead, 2005). This upper bound is equal to sqrt(rel1 * rel2). Therefore, by using measurements with poor reliability, one may overlook true correlations even when they are strong and robust in reality. Importantly, low reliability also affects effect sizes and replicability of the results; therefore, it deserves even more attention in experimental psychology (Green et al., 2016). At the same time, the issue of reliability has been largely neglected in experimental psychology (Maloney et al., 2010). This may be partly due to reasons discussed recently by Hedge and colleagues (2018). Accordingly, the inherent goal of experimental psychology is to trace robust and reproducible cognitive phenomena. Reaching this goal seems more straightforward if a given phenomenon is apparent across all individuals and when there is no major variation between participants within a sample. Such homogeneity across participants is, however, not ideal when it comes to estimating reliability. The determination of reliability in classical psychometric test theory involves correlations; it not only depends on measurement error but also on between-subject variance ${ }^{1}$. Reliability is high when the values or the rank order of the subjects obtained with the test on occasion 1 (e.g., measured with parallel version of the test, one half of the test ${ }^{2}$ or one administration of the test) are highly correlated to the values or rank order obtained with the same test at occasion 2. Reliability can be influenced by both task and sample characteristics. It decreases not only for larger error variance, whilst holding inter-individual variance constant, but

\footnotetext{
${ }^{1}$ Another way of establishing reliability going beyond the classic psychometric test theory considers using intraclass correlations, which can be better conceptualized in terms of generalizability theory and can take into account absolute agreement of scores (see Hedge et al., for examples of use). ${ }^{2}$ Cronbach's alpha, which is a popular method to measure internal consistency of psychometric tests, can be expressed as a combination of all possible split-half reliabilities.
} 
also for smaller inter-individual variance, whilst holding error variance constant. Reliability is thus likely lower in homogeneous samples usually required in experimental psychology for the assessment of robust cognitive effects. As a consequence, using the reliability of a test as an index for its measurement error strongly depends on sample characteristics (see also Martin \& Rast, 2020). Therefore, it is not (always) suited for the investigation of the prevalence of cognitive effects at the individual level. Needless to say, presence of a significant effect at the group level does not imply that it is uniformly present in all individuals (see e.g., Klaassen, 2020).

This problem was discussed recently by Luck (2019), who, as a solution, differentiated between measurement reliability and measurement precision. Luck defined measurement precision as an index for the stability of the measurement within an individual. In many psychological studies, several data-points are collected for each experimental condition for each participant. This allows for the calculation of the stability of the differences between conditions, depending on which trials are taken for averaging. When a task is measured with high precision, these differences are relatively stable. Where both measurement reliability and precision give an idea about the quality (and/ or stability) of the data, the calculation of measurement precision can be performed independently from the sample being tested and can be estimated for each participant separately. To our knowledge, the estimation of measurement precision is not common practice in experimental and differential psychology, and techniques to determine the precision of a measurement are not (widely) available. Interestingly, having an estimate of the precision of an individual's test score can be an alternative to quantify whether a cognitive effect is significantly present at the level of the individual participant or not. 
The nature of cognitive phenomena, or more precisely, whether they are present in all individuals or whether there are qualitative individual differences, was intensely investigated by Haaf and Rouder (Haaf et al., 2018; Haaf \& Rouder, 2019; Rouder et al., 2019; Rouder \& Haaf, 2020). They propose an original approach based on Bayesian modeling to answer this specific question. They show that while some effects, such as the Stroop effect, seem to be present in each individual, in some other phenomena, such as the Simon effect, there are qualitative individual differences (Haaf \& Rouder, 2017). Their approach is very promising and still in development. Nevertheless, at this stage, it is applicable to experiments in which two conditions are compared, and it does not allow extensions to more complex indices of performance. At the same time, in its current form, it only allows for the determination of whether there are significant individual differences between conditions, not whether an individual shows a (cognitive) effect in a reliable manner. This is reminiscent of the meaning of a significant random effect for the factor "participant" in linear mixed models (Brown, 2020; Meteyard \& Davies, 2020). Again, this statistical method is only able to determine the presence of significant individual differences between conditions and does not provide information about whether a given participant reveals a reliable nonzero effect. In other words, the field is still in need of a (statistical) tool to quantify whether a cognitive effect is significantly present at the level of the individual participant or not.

\section{Quantifying cognitive effects at the individual level}

Considering that the reliability of robust and reproducible cognitive phenomena is usually far from perfect, this inaccuracy needs to be taken into account when deciding whether an individual reveals a certain cognitive effect or not. One of the ways to 
overcome this problem is by applying statistical inference procedures within each participant (see e.g., Little et al., 2011; Onghena, 2020; Thiele et al., 2017). Another possibility normally adhered to in classical test theory - but so far rarely in experimental psychology - is to deal with the issue of low reliability and calculate a confidence interval $(\mathrm{Cl})$ around the true score. The idea is that each measurement is comprised of an underlying true score and a measurement error randomly distributed around it. Usually, these errors are assumed to be normally distributed. Calculation of the $\mathrm{Cl}$ can be conducted using different approaches which we will describe below.

Psychometric approach. In the psychometric approach, Cls are calculated by means of accounting for measurement error. More concretely, the reliability of the measurement as well as the standard deviation (SD) of scores in the sample are considered to estimate the standard error of measurement (SEM) as follows: SEM = $S D(X){ }^{*}[\operatorname{sqrt}(1-$ reliability $(X))]$. Assuming a normal distribution for $X$, the SEM is then taken to provide a $(1-\alpha) \mathrm{Cl}$ around the individual observed score: $95 \% \mathrm{Cl}=\mathrm{X} \pm(1.96$ * SEM), e.g., for $\alpha=0.05$. Unfortunately, this approach suffers from a number of limitations. First, it can only be used if the sample meets a fundamental homogeneity assumption. Namely, the SEM is assumed to be constant across all members of the population from which the sample is drawn to compute the reliability estimate (cf. e.g., Willmes, 2010). Second, although the psychometric approach accounts for measurement error (i.e., task characteristics), it does not solve the problem of dependence on sample characteristics (i.e., the $\mathrm{Cl}$ is assumed to be the same for each participant, irrespective of whether their response pattern is characterized by small or large variation), a problem frequently criticized from the perspective of probabilistic test theory (Crocker \& Algina, 1986). A possible solution to this problem is to estimate the reliability and SD from a larger representative sample of healthy individuals or another 
population of interest to get relatively precise and stable estimates, but this is not ideal, as Cls should be determined based on the stability of the behavioral response of an individual, without taking recourse to the performance of the other participants of the experiment ${ }^{3}$. This is possible within the bootstrapping approaches discussed below.

Bootstrapping approaches. In the bootstrapping approaches, a random sample of trials responded to by a given participant is selected to calculate the effect of interest. This procedure of random sampling (with replacement) is repeated numerous times (usually a few thousand) to obtain a distribution of estimates for the effect (for a sample use in numerical cognition see Crollen \& Noël, 2015; for a comprehensive introduction to bootstrap techniques and its usefulness in psychology, see Rousselet et al., 2019). The range in which a certain proportion of these estimates $(1-\alpha)$ is located is subsequently determined by removing the lowest $\alpha / 2$ and highest $\alpha / 2$ fraction of the distribution and can be taken to be the participant's $\mathrm{Cl}$. This method will be referred to as $\mathrm{H} 1$ bootstrapping, where $\mathrm{H} 1$ stands for "alternative hypothesis", because it tests for the robustness of an effect depending on the trials being selected for averaging. As an alternative, random sampling with replacement can be used to allocate trials from different experimental conditions to two sets (such a logic to some extent, resembles the specification of the distribution under the null hypothesis method proposed by Simonsohn et al., 2020). This mathematical procedure reflects the null hypothesis model perfectly (i.e., samples for both "conditions" to be compared are drawn from the same pool). Subsequently, it is tested whether the empirically observed difference between conditions is likely to be observed under the null hypothesis model. If the empirical difference is located outside the "middle" 1- $\alpha$ proportion of the distribution of

\footnotetext{
${ }^{3}$ Haaf and Rouder (2017; Appendix A) propose a frequentist method for calculating individual confidence intervals however their method is only suited for designs in which only two conditions are compared.
} 
resampled differences, one might conclude with 1 - $\alpha$ confidence that the empirically observed difference is unlikely under $\mathrm{H} 0$. This approach can therefore be referred to as $\mathrm{HO}$ bootstrapping, where $\mathrm{HO}$ stands for "null hypothesis". Crucially, with these bootstrapping methods, the breadth of the $\mathrm{Cl}$ may be different for each participant. The $\mathrm{Cl}$ depends only on the stability of the behavioral response within the participant and is not influenced by the performance of other participants like in the psychometric approach. For this reason, the bootstrap $\mathrm{Cl}$, and especially the width of the $\mathrm{Cl}$, could indicate the measurement precision (Luck, 2019). Consequently, this feature makes the bootstrapping approach ideally suited for estimating prevalence.

Current approach - uniform analysis of multiple existing data sets: strengths and weaknesses

Scientific progress does not only depend upon the reliability of the measurements; true insight in psychological processes and mechanisms should be based on highly replicable observations. In psychology, such replications are not always obvious, and today, several researchers argue that psychology suffers from a replication crisis (e.g., Chambers, 2017; Pashler \& Wagenmakers, 2012). Multiple classical findings in different psychological domains were indeed very difficult to replicate, even when researchers used the exact experimental setup and sample characteristics as in the original study (for an example in the domain of numerical cognition, see Colling et al., 2020). Several reasons for this crisis have been put forward (e.g., underpowered studies, small effect sizes, low reliability Button et al., 2013; Maxwell et al., 2015; Shrout \& Rodgers, 2018). As a solution, meta-analytic and multi-lab approaches are typically proposed where conclusions are based on the results of a series of (highly) comparable studies conducted on different samples. 
In the current study, we propose a novel approach that is situated between a typical meta-analytic approach and multi-lab initiatives, and thus shares some of the advantages of both approaches. On the one hand, the typical meta-analytic approach is based on extracting and comparing effect sizes from several (un)published studies. A big advantage of this approach is that it is possible to take into account data coming from an extraordinarily high number of participants without requiring costly and effortful (novel) data collection. However, in most cases, researchers need to deal with aggregated data and only have the statistics reported by the authors of the original studies at their disposal, which does not allow them to control for differences in experimental designs, methods of data preprocessing, outlier exclusion, and analyses. These aspects, as well as the statistics actually reported in the primary studies, might differ considerably between studies, which makes direct comparisons less straightforward. Thorough control for these parameters requires them to be orthogonal between studies, which is often not the case. For instance, some labs routinely use certain task parameters and data preprocessing routines, whereas other labs consistently use other configurations. Therefore, it can be difficult to determine the source of potential discrepancies between results (see Botvinik-Nezer et al., 2020; and Silberzahn et al., 2018 for extreme examples). On the other hand, multi-lab initiatives (e.g., LeBel, 2015) are based on data collection using exactly the same task, data preprocessing, and analyses, but this approach requires new data collection, which is often time consuming and costly. Within this multi-lab approach, it is also possible to systematically manipulate different methodological configurations to see their influence on the observed effects. Because new data needs to be collected, sample sizes (and number of experiments considered) are usually smaller than in typical meta-analyses.

The approach proposed here is a combination of these two and is particularly useful for phenomena that have been investigated in a relatively similar manner across labs, 
which is the case for many cognitive effects (for a similar approach, see Schnuerch et al., 2020). In line with the meta-analytic approach, it is based on considerable sample sizes without requiring new data collection, and, as for multi-lab initiatives, it allows for an in-depth analysis starting from the raw data, thereby avoiding differences in data preprocessing and statistical reporting. Importantly, the availability of data from multiple studies makes it possible to determine the heterogeneity of the observed effects across the included data sets (a common approach in meta-analyses; e.g., Higgins et al., 2020). From these results, conclusions can be drawn about the robustness and replicability of the findings. Compared to these advantages, the drawbacks remain quite limited (e.g., fewer studies than for typical meta-analyses and no possibility for controlling or unifying task parameters, which is possible in multi-lab studies).

The method we propose resembles the mega-analysis approach proposed within fMRI research (Costafreda, 2009), and, to some extent, the integrative data analysis (IDA; Curran \& Hussong, 2009; Hussong et al., 2013) approach (see also Weston et al., 2020). Within the IDA framework, raw data from multiple studies are put together and analyzed. Typically, the IDA framework assumes running meta-analyses, which is based on participant level data and accounts for the effect of the study (treating it as either a fixed or a random effect), rather than relying on the study level summary statistics. However, it is not typical for the IDA framework to go down to the single trial level (H. Cooper \& Patall, 2009). Such an approach, while popular in health and clinical psychology, has not gained much popularity in experimental (cognitive) psychology, despite obvious benefits.

To sum up, the present approach provides a useful methodology, especially in light of the recently discussed "replicability crisis" in psychology because it allows optimal data reuse and making conclusions based on samples considerably larger than ones 
that are feasible to collect within a single study. Although it cannot replace metaanalyses or multi-lab initiatives, it can provide highly interesting complementary evidence.

\section{Taking the SNARC effect as an example}

To demonstrate this novel approach and how the bootstrapping methods can be used to determine the absence/presence of cognitive effects at the individual level, this study focuses on the so-called Spatial Numerical Association of Response Codes (SNARC) effect (Dehaene et al., 1993). The SNARC effect reflects the observation that, in binary-choice reaction time (RT) tasks on numbers, participants from Western cultures respond faster to small numbers with left-sided response keys and to large numbers with right-sided response keys. This effect was first documented over 25 years ago and has become one of the most thoroughly investigated phenomena in the domain of numerical cognition (the original paper by Dehaene and colleagues has been cited over 3000 times, Google Scholar search reveals over 450 papers with the term "SNARC" in the title). The typical task for measuring the SNARC effect is a bimanual parity judgment where participants decide whether a given number is odd or even with a left or right key press. The response mapping is reversed halfway through the experiment so that left- and right-sided responses are collected for each number in every participant. The SNARC effect is typically attributed to the spatial mental representation of numerical magnitude within a particular cultural and linguistic context (e.g., Dehaene et al., 1993; Shaki et al., 2009). Some nativist accounts have also been proposed (e.g., de Hevia et al., 2017; Rugani et al., 2015) as well as ones based on conceptual coding (Gevers et al., 2010) or spatial coding in working memory (Fias \& van Dijck, 2016). In this last view, small numbers would be temporarily mentally 
represented on the left side and large numbers on the right side of a visuo-spatial medium taking the form of a mental number line.

The SNARC effect has not only been replicated in many studies using parity judgment (see Cipora et al., 2019 for a large-scale online replication) and other experimental paradigms (Cipora et al., 2018 for an overview). It has also been reported in various other numerical tasks in both typical and atypical populations, across various developmental stages (for reviews see Fischer \& Shaki, 2014; Toomarian \& Hubbard, 2018; for a metaanalysis, see Wood et al., 2008), generally highlighting robustness of the effect. Interestingly, despite its robustness at the group level, the SNARC effect is characterized by high inter-individual variability (for early investigations, see Wood et al., 2006b), which can be accounted for by multiple factors (Cipora et al., 2020). As also documented in earlier literature (e.g., Wood et al., 2006a), across several studies, it was consistently reported to be present in about $70-85 \%$ of participants (see Table 1 for details and relevant references). 
Table 1. Overview of previous studies on the SNARC effect considering individual differences. Only studies considering healthy adult participants included.

\begin{tabular}{|c|c|c|c|c|c|c|c|c|c|}
\hline Article & $\begin{array}{l}\text { Experiment / } \\
\text { Group }\end{array}$ & $\mathrm{n}$ & $\begin{array}{l}\text { Number } \\
\text { range }\end{array}$ & $\begin{array}{l}\text { Mean } \\
\text { slope } \\
(\mathrm{SD})^{\mathrm{a}}\end{array}$ & $\begin{array}{l}\mathrm{n} \\
\text { slopes } \\
<0\end{array}$ & $\begin{array}{l}\% \\
\text { slopes } \\
<0\end{array}$ & $\begin{array}{l}\mathrm{n} \\
\text { slopes } \\
>0\end{array}$ & Comments & $\begin{array}{l}\text { SNARC } \\
\text { effect } \\
\text { reliability }\end{array}$ \\
\hline \multirow{2}{*}{$\begin{array}{l}\text { Hoffmann, } \\
\text { Pigat, and } \\
\text { Schiltz (2014) }\end{array}$} & Young adults & 28 & $0-9$ & $-7.78(\mathrm{NR})$ & 25 & 89.3 & 3 & \multirow[b]{2}{*}{ Data retrieved from their Fig. $2 a$} & - \\
\hline & Older adults & 54 & $0-9$ & $\begin{array}{l}-15.48 \\
(\mathrm{NR})\end{array}$ & 51 & 94.4 & 3 & & - \\
\hline \multirow{3}{*}{$\begin{array}{l}\text { Hoffmann, } \\
\text { Mussolin, } \\
\text { Martin, and } \\
\text { Schiltz (2014) }\end{array}$} & Control & 38 & $0-9$ & $-8.82(\mathrm{NR})$ & 34 & 89.5 & 4 & - & - \\
\hline & Math expert & 38 & $0-9$ & $-5.25(\mathrm{NR})$ & 10 & 26.3 & 28 & - & - \\
\hline & Math difficulty & 19 & $0-9$ & $\begin{array}{c}-13.23 \\
(\mathrm{NR})\end{array}$ & 19 & 100.0 & 0 & - & - \\
\hline Fischer (2008) & Exp. 2 & 100 & $\begin{array}{l}1-8 \text { or } \\
1,2,8,9\end{array}$ & $\begin{array}{l}-6.86 \\
(12.31)\end{array}$ & 76 & 76.0 & 24 & $\begin{array}{l}\text { Approximated data based on } \\
\text { their Fig. 2; Number range } \\
\text { differed between participants }\end{array}$ & - \\
\hline $\begin{array}{l}\text { Shaki and } \\
\text { Fischer (2008) }\end{array}$ & Exp. 1 & 18 & $1-4 ; 6-9$ & $-9.90(N R)$ & 14 & 77.8 & 4 & $\begin{array}{l}\text { Data retrieved from their Fig. 1, } \\
\text { the left-to-right reading } \\
\text { condition }\end{array}$ & - \\
\hline $\begin{array}{l}\text { Shaki, } \\
\text { Fischer, and } \\
\text { Petrusic } \\
\text { (2009) }\end{array}$ & $\begin{array}{l}\text { Canadian } \\
\text { participants }\end{array}$ & 12 & $1-4 ; 6-9$ & $\begin{array}{l}-10.81 \\
(12.12)\end{array}$ & 10 & 83.3 & 2 & 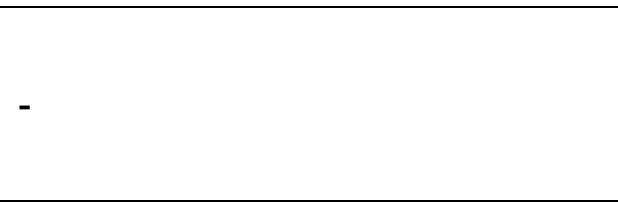 & - \\
\hline \multirow{2}{*}{$\begin{array}{l}\text { Fias, } \\
\text { Lauwereyns, } \\
\text { and } \\
\text { Lammertyn } \\
(2001)\end{array}$} & Exp. 1 & 24 & $0-9$ & $-2.03(3.4)$ & 17 & 70.8 & 7 & $\begin{array}{l}\text { Task referred to orientation of } \\
\text { triangle superimposed on } \\
\text { number }\end{array}$ & - \\
\hline & Exp. 4 & 23 & $0-9$ & $-3.74(5.0)$ & 17 & 73.9 & 6 & $\begin{array}{l}\text { Task referred to orientation of a } \\
\text { line placed next to number }\end{array}$ & - \\
\hline \multirow{3}{*}{$\begin{array}{l}\text { Viarouge, } \\
\text { Hubbard, and } \\
\text { McCandliss } \\
(2014)\end{array}$} & Average & 35 & $1-4 ; 6-9$ & NR (NR) & 28 & 80.0 & 7 & Data retrieved from their Fig. 4 & $.71^{\mathrm{c}}$ \\
\hline & Session 1 & 35 & $1-4 ; 6-9$ & $\mathrm{NR}(\mathrm{NR})$ & 27 & 77.1 & 8 & \multirow{2}{*}{ Data retrieved from their Fig. 3} & - \\
\hline & Session 2 & 35 & $1-4 ; 6-9$ & $N R(N R)$ & 26 & 74.3 & 9 & & \\
\hline
\end{tabular}




\begin{tabular}{|c|c|c|c|c|c|c|c|c|c|}
\hline $\begin{array}{l}\text { Fattorini, } \\
\text { Pinto, } \\
\text { Rotondaro, } \\
\text { and Doricchi } \\
(2015)\end{array}$ & Exp. 1 & 60 & $1-4 ; 6-9$ & $-9.4(9.1)$ & 53 & 88.3 & 7 & $\begin{array}{l}\text { Data retrieved from their Fig. } 2 \\
\text { panel C }\end{array}$ & .75 \\
\hline $\begin{array}{l}\text { Pinhas, Shaki, } \\
\text { and Fischer } \\
(2014)\end{array}$ & Exp. 1 & 54 & $1,2,8,9$ & $\begin{array}{c}-8.72 \\
(10.73)\end{array}$ & 44 & 81.5 & 10 & Data retrieved from their Fig. 3 & - \\
\hline Fabbri(2013) & $\begin{array}{l}\text { Italian } \\
\text { participants }\end{array}$ & 48 & $1-4 ; 6-9$ & $\begin{array}{l}-5.79 \\
(9.06)\end{array}$ & 38 & 79.2 & 10 & $\begin{array}{l}\text { Data retrieved from their Table } \\
3 \text {, averaged across left- and } \\
\text { right starters in the horizontal } \\
\text { parity task }\end{array}$ & \\
\hline $\begin{array}{l}\text { Schwarz \& } \\
\text { Mueller (2006) }\end{array}$ & Exp. 2 & 23 & $0-9$ & $\begin{array}{l}-4.36 \\
(4.94) \\
\end{array}$ & 20 & 87.0 & 3 & $\begin{array}{l}\text { Bimanual condition. Data } \\
\text { retrieved from their Fig. } 3\end{array}$ & - \\
\hline $\begin{array}{l}\text { van Dijck and } \\
\text { Fias (2011) }\end{array}$ & Exp. 2. & 36 & $1-6$ & $-9.05(\mathrm{NR})$ & 27 & 75.0 & 9 & - & - \\
\hline $\begin{array}{l}\text { Jonas, Spiller, } \\
\text { Jansari, and } \\
\text { Ward (2014) }\end{array}$ & $\begin{array}{l}\text { Exp. 1, non- } \\
\text { synaesthetes } \\
\text { only }\end{array}$ & 27 & $1-9$ & $\begin{array}{c}-1.83 \\
(7.50)[\mathrm{ns}]\end{array}$ & 16 & 59.3 & 11 & $\begin{array}{l}\text { Data retrieved from their } \\
\text { supplementary Table } 1 \text { d }\end{array}$ & - \\
\hline $\begin{array}{l}\text { Yang et al. } \\
(2014)\end{array}$ & Adults & 42 & $1-9$ & $\begin{array}{l}-6.07 \\
(9.27) \\
\end{array}$ & 35 & 83.3 & 7 & $\begin{array}{l}\text { Data retrieved from their Table } \\
4\end{array}$ & - \\
\hline \multirow{3}{*}{$\begin{array}{l}\text { Ninaus et al. } \\
(2017)\end{array}$} & Young adults & 25 & $1,2,8,9$ & $-10(7)$ & 21 & 84.0 & 4 & \multirow{3}{*}{$\begin{array}{l}\text { Reliabilities calculated based } \\
\text { on standardized reaction times }\end{array}$} & .93 \\
\hline & $\begin{array}{l}\text { Middle-aged } \\
\text { adults }\end{array}$ & 27 & $1,2,8,9$ & $-10(10)$ & 23 & 85.2 & 4 & & .95 \\
\hline & Elderly & 24 & $1,2,8,9$ & $-16(12)$ & 22 & 91.7 & 2 & & .96 \\
\hline $\begin{array}{l}\text { Cipora, } \\
\text { Soltanlou, } \\
\text { Reips, and } \\
\text { Nuerk (2019) }\end{array}$ & - & 1056 & $1-4 ; 6-9$ & $\begin{array}{c}-8.49 \\
(10.28)\end{array}$ & 877 & 83 & 179 & Online experiment & .43 \\
\hline \multicolumn{2}{|l|}{ OVERALL } & 1881 & - & - & 1530 & 81.31 & 351 & $\begin{array}{l}\text { Weighted average, } \\
\text { considering sample size; }\end{array}$ & - \\
\hline
\end{tabular}




\begin{tabular}{|l|l|l|l|l|l|l|l|}
\hline & & & & & & & unweighted average = \\
$\mathbf{7 9 . 5 9 \%}$
\end{tabular}

a Number of decimals reported as in the original studies, hence inconsistent between rows of the table (NR = not reported)

b Split-half, Spearman-Brown corrected (only if reported)

${ }^{c}$ Calculated based on .545 raw correlation between half-based slopes from both sessions collapsed, without excluding outliers, as reported by the authors, adjusted with Spearman-Brown correction.

d Data from supplementary Table 1 diverges from results reported in the main text as regards sample size and significance of the SNARC effect at the group level 
This rough prevalence estimate is based on the most common method of quantifying the effect (Fias et al., 1996; Lorch \& Myers, 1990) using individual slope estimates. First, for each participant separately, RT differences (dRTs) are calculated for each number separately by subtracting the average (or median) RT of the left hand from the average (or median) RT of the right hand. Consequently, negative dRTs indicate an advantage of right-handed responses as compared to left-handed responses. Second, for each participant separately, dRTs are regressed on their corresponding number magnitude employing the least squares method. The obtained unstandardized regression coefficient (or slope) is taken as a measure of the participant's SNARC effect. It is expressed in milliseconds and can be interpreted as the increase in righthanded RT advantage compared to the left-handed RT when the magnitude of the number increases by one. Accordingly, a more negative slope indicates a stronger SNARC effect; conversely, positive slopes indicate that the right-hand advantage decreases with increasing number magnitude, which reflects a reverse SNARC effect. Third, to test whether a significant SNARC effect is observed at the group level, the sample of individual slope estimates is then tested against zero by means of a onesample $t$-test.

Even though this method is commonly employed to quantify the SNARC effect, the use of unstandardized slopes has been criticized. First, they do not consider the fit of the regression model (Pinhas et al., 2012). The SNARC effect is actually one of the few instances where a raw slope measure is deemed more important in many papers (including some of our own) than explained variance or goodness of fit of the regression model (for a similar argument see also Tzelgov et al., 2013). Moreover, unstandardized slopes are very sensitive to a participant's mean RT and to intraindividual variability in RT. Namely, there is a very high correlation between mean RT and SD(RT), usually above .8 (e.g., Cipora et al., 2016; Cipora \& Nuerk, 2013). This 
means that values of all differential measures calculated in absolute units (milliseconds) become larger due to higher variance. Such an influence of larger general variability can be avoided by using standardized slopes. They can be easily obtained from the same individual regression analysis as the unstandardized ones. In case of a statistical model with one single regressor, the standardized slope is equal to the Pearson correlation between number magnitude and dRT. To approximate a normal distribution, standardized slopes are typically Fisher z-transformed. Using standardized measures rather than unstandardized ones may thus provide a more valid measure of the SNARC effect (Lyons et al., 2015).

To determine whether an individual participant demonstrates a SNARC effect, one typically simply checks whether the unstandardized or standardized slope is larger or smaller than 0 . About 70 to $80 \%$ of participants usually exhibit slopes below 0 and are therefore considered as revealing the effect (cf. Table 1). However, as already discussed, this approach is too simplistic as it does not consider that a participant's slope is just an estimate of the true slope parameter combined with measurement error. Classifying participants based on whether their SNARC slope is larger or smaller than 0 would only be valid if the slope is perfectly estimated without measurement error. In the psychometric model of classical test theory, this would only be true if the reliability of the SNARC effect equals 1 . Studies estimating the reliability of the SNARC effect, however, indicate that this is clearly not the case; rather, reliability varies from low to very high (Tables 1 and 3; see Appendix A for an algorithm for estimating the reliability of the SNARC effect). This inaccuracy thus necessitates the calculation of Cls around the observed slope to quantify the SNARC effect at the individual level. Note that one can think of two different types of $\mathrm{Cl}$ : one around an observed score of a given participant, and one around the sample mean. Here we consider the first perspective. Using the psychometric approach and assuming a normal distribution, the $\mathrm{Cl}$ around 
the estimated slope $x^{*}$ is computed as follows: $95 \% \mathrm{Cl}=\mathrm{x}^{*} \pm(1.96 *$ SEM), where SEM is given by $S D(x){ }^{*}[\operatorname{sqrt}(1-$ reliability $(x))]$. If the $\mathrm{Cl}$ does not contain 0 and the slope is negative, one may claim with predefined confidence that the participant reveals a reliable SNARC effect. Alternatively, if the $\mathrm{CI}$ does not contain 0 and the slope is positive, one may claim that the participant reveals a reliable reversed SNARC effect. Finally, if the $\mathrm{Cl}$ contains 0 , the participant should be classified as not revealing a reliable SNARC effect. As explained above, this approach starts from the assumption that the $\mathrm{Cl}$ is equal for all participants within a sample. This assumption is, however, often violated. As a solution, we propose the use of two different bootstrapping methods.

In the bootstrapping $\mathrm{H} 1$ approach, a participant is considered to reveal a reliable SNARC effect if a negative slope is observed, irrespective of the random sample of RTs used to estimate the slope. More precisely, for each participant within each experimental cell (number $\times$ response hand), a random sample of trials can be selected (with replacement) and used to calculate the SNARC slope across the random samples from all numbers. This procedure is repeated a few thousand times to obtain the participant's $\mathrm{Cl}$. The presence of a reliable SNARC effect at a certain $(1-\alpha)$ confidence level is then again checked by examining whether the $\mathrm{Cl}$ contains 0 .

In the bootstrapping $\mathrm{H} 0$ approach, we mathematically create a situation where the null hypothesis is true, that is, where there is no link between numerical magnitude and response side. For each participant, two sets of responses are sampled with replacement from all (right-handed and left-handed) RTs to a given number. One of these sets is treated as "left-handed responses" and the other as "right-handed responses". Subsequently, the SNARC slope is calculated. After repeating this procedure multiple times, the distribution of the resulting slopes is compared with the empirically observed slope. If the latter is located outside the $(1-\alpha) \mathrm{Cl}$, one concludes 
with 1 - $\alpha$ confidence that the empirically observed slope is unlikely to be under $\mathrm{HO}$, that is, when there is no link between magnitude and response side.

\section{Aim of the present study}

In the current study we demonstrate the usefulness of the psychometric and bootstrapping approaches to determine the prevalence of the SNARC effect at the individual level. To ensure robust conclusions, we conducted a series of uniform analyses on 18 different data sets (published and unpublished) collected by different labs across Europe using the typical bimanual parity judgment task. For each study, we separately calculated standardized and unstandardized regression slopes as quantifications of the SNARC effect, determined Cls based on both the psychometric and bootstrapping methods, and calculated the proportion of participants revealing a reliable SNARC effect according to both methods. The resulting proportions were then compared to the prevalence of the SNARC effect previously reported in the literature where only single SNARC slope estimates had been considered (see Table 1).

Alongside this main goal, we also assessed which sample and task parameters might influence the proportion of participants revealing a reliable SNARC effect. Finally, we conducted additional analyses to investigate whether participants' age and gender as well as block order in the parity task affect the observed SNARC effect (see Appendices B \& C).

\section{METHODS}

Included data sets 
Data from 18 studies conducted in eight different labs in five different countries were included in the present analysis ${ }^{4}$, with a total of 1016 participants (635 females and 361 males; for 20 participants, gender data was not available) $)^{5}$. Information about each data set and its sample characteristics are summarized in Table 2 and presented in more detail in an accompanying data paper (Cipora et al., 2022), which also provides detailed documentation of variables and prospects for future data reuse. Only studies utilizing bimanual parity judgment of single-digit numbers administered to healthy adults (age range 17-81 years) were considered. In one study (Georges et al., 2018), participants met the criteria of ADHD diagnosis. Thirteen studies used numbers 1 to 4 and 6 to 9 , while five studies used all single-digit numbers from 0 to 9 ; for the sake of comparability between data sets, numbers 0 and 5 were excluded post-hoc from the latter before any other operations were performed on the data. Both blocks of hand-toparity assignment were administered immediately one after the other, except in Nuerk et al. (2005), where parity judgments on numbers presented in different formats (e.g., auditory, dice patterns, etc.) were intermixed. In 14 studies, the order of blocks was counterbalanced between participants; in the remaining four studies, it was fixed. Response-to-key assignment changed only once in the middle of the experiment ${ }^{6}$. Additionally, in van Dijck et al. (unpublished a), $50 \%$ of the trials were presented with upright digits and $50 \%$ in italics for the sake of consistency with other tasks.

\footnotetext{
${ }^{4}$ Raw data sets are available at https://osf.io/n7szg/

${ }^{5}$ In Appendix B we present the analyses of gender and age differences in the SNARC effect.

${ }^{6}$ Block order did not influence the SNARC effect. See Appendix C for details.
} 
Table 2. Overview of analyzed data sets.

\begin{tabular}{|c|c|c|c|c|c|c|c|c|c|}
\hline \multirow[t]{2}{*}{ Data set } & \multirow{2}{*}{$\begin{array}{l}\text { Number } \\
\text { range }\end{array}$} & \multirow{2}{*}{$\begin{array}{l}\text { Block } \\
\text { order } \\
\text { a }\end{array}$} & \multirow{2}{*}{$\begin{array}{l}\text { Blocks } \\
\text { immediatel } \\
\text { y one after } \\
\text { another }\end{array}$} & \multicolumn{6}{|c|}{ Sample description } \\
\hline & & & & $\mathrm{N}$ & $\begin{array}{l}\text { Age } \\
\text { (SD) }\end{array}$ & $\begin{array}{l}\text { Gender } \\
(\mathrm{f} / \mathrm{m})\end{array}$ & Mother tongue & Background & Comments \\
\hline $\begin{array}{l}\text { Cipora \& Göbel } \\
(2013)\end{array}$ & $0-9$ & C & yes & 51 & $\begin{array}{l}23.2 \\
(4.9)\end{array}$ & $30 / 21$ & English & $\begin{array}{l}\text { Students from the } \\
\text { University of York }\end{array}$ & $\begin{array}{l}\text { no } \\
\text { restrictions }\end{array}$ \\
\hline $\begin{array}{l}\text { Cipora et al. } \\
(2016)\end{array}$ & $1-4 ; 6-9$ & $\mathrm{C}$ & yes & 44 & $\begin{array}{l}27.9 \\
(1.1)\end{array}$ & $6 / 38$ & Polish & $\begin{array}{l}\text { Advanced doctoral } \\
\text { students: math, } \\
\text { engineering, humanities } \\
\text { and social sciences }\end{array}$ & $\begin{array}{l}\text { no physics } \\
\text { students }\end{array}$ \\
\hline Cipora (2014) & $1-4 ; 6-9$ & C & yes & 55 & $\begin{array}{l}23.1 \\
(3.7)\end{array}$ & $39 / 16$ & Polish & General population & $\begin{array}{l}\text { no } \\
\text { psycholog } \\
\text { y students }\end{array}$ \\
\hline $\begin{array}{l}\text { Georges et al. } \\
(2017)\end{array}$ & $1-4 ; 6-9$ & $f$ & yes & $81^{b}$ & $\begin{array}{l}23.4 \\
(3.2)\end{array}$ & $40 / 41$ & $\begin{array}{l}\text { heterogeneou } \\
\mathrm{s}\end{array}$ & $\begin{array}{l}\text { Students from University } \\
\text { of Luxembourg }\end{array}$ & $\begin{array}{l}\text { no } \\
\text { psycholog } \\
\text { y students }\end{array}$ \\
\hline $\begin{array}{l}\text { Georges et al. } \\
(2018)\end{array}$ & $1-4 ; 6-9$ & $f$ & yes & 26 & $\begin{array}{l}26.9 \\
(3.3)\end{array}$ & $15 / 11$ & $\begin{array}{l}\text { heterogeneou } \\
\mathrm{s}\end{array}$ & $\begin{array}{l}\text { Students from University } \\
\text { of Luxembourg; meeting } \\
\text { diagnostic criteria of } \\
\text { ADHD }\end{array}$ & $\begin{array}{l}\text { no } \\
\text { psycholog } \\
\text { y students }\end{array}$ \\
\hline $\begin{array}{l}\text { Georges et al. } \\
\text { (unpubl.) }\end{array}$ & $1-4 ; 6-9$ & $f$ & yes & 55 & $\begin{array}{l}24.8 \\
(3.4) \\
\end{array}$ & $35 / 20$ & $\begin{array}{l}\text { heterogeneou } \\
\mathrm{s}\end{array}$ & $\begin{array}{l}\text { Students from University } \\
\text { of Luxembourg }\end{array}$ & $\begin{array}{l}\text { no } \\
\text { restrictions }\end{array}$ \\
\hline
\end{tabular}




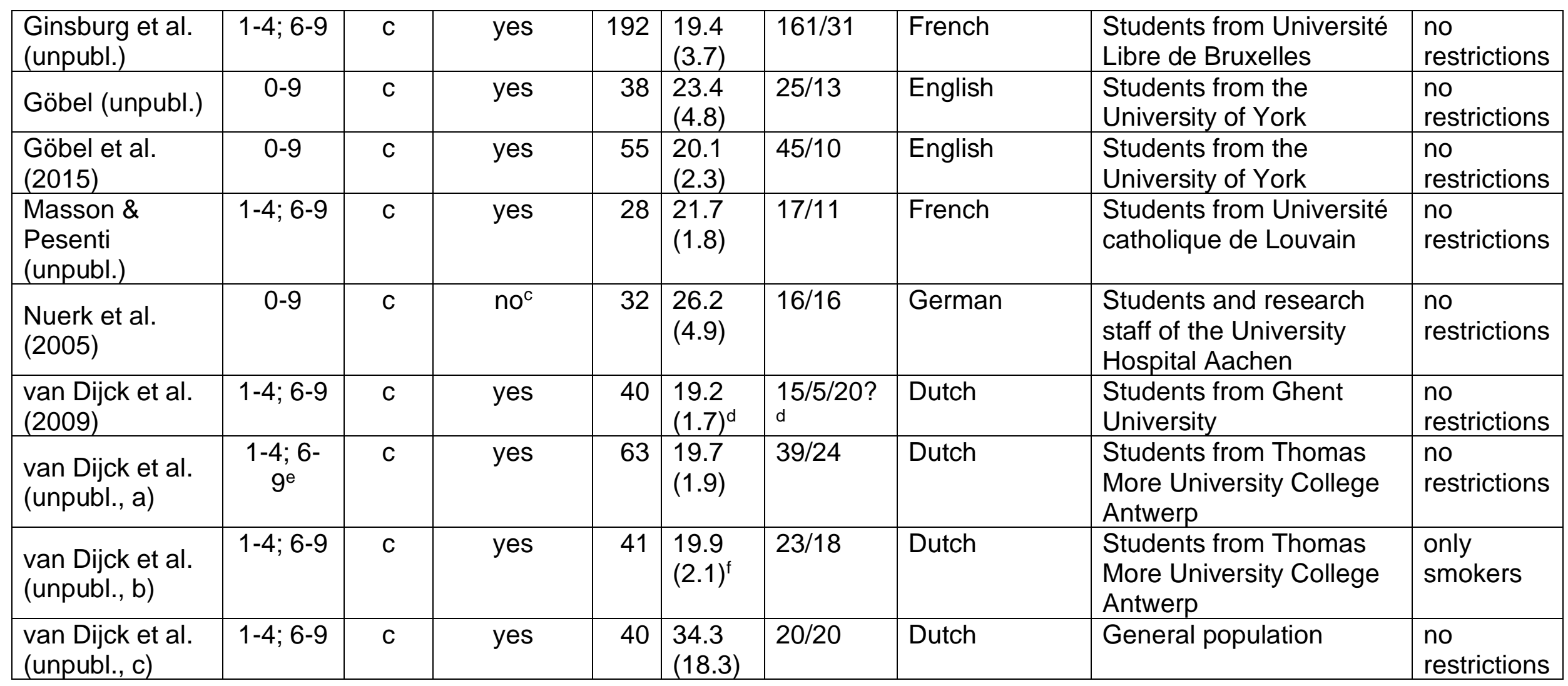

${ }^{\text {a }} \mathrm{c}=$ counterbalanced between participants; $f=$ fixed

$\mathrm{b}$ three participants were administered with more trials than the rest of the participants

c blocks were intermixed with other binary decision tasks, e.g., parity judgment of numbers presented in another modality

d demographic data of 20 participants was not available any more

e numbers presented upright or in italics for consistency with other blocks

${ }^{f}$ age data from 2 participants were missing 


\section{Analyses}

Individual trial data from each data set were processed, and the SNARC effect slopes were computed using the following protocol${ }^{7}$. First, practice sessions and incorrect responses were discarded. Second, outlier RTs were removed. In the first step, correct responses less than 200 ms were treated as anticipations and discarded. Subsequently, a sequential trimming method was applied. Mean RT and $\mathrm{SD}(\mathrm{RT})$ were calculated for each participant separately, and correct RTs outside \pm 3 SD from an individual's mean RT were discarded. This procedure was repeated until means and SDs no longer changed. Correct RTs, which were retained after trimming, were used for further analysis. The proportion of RTs included in the analysis for each data set is reported in Table 3. Third, unstandardized and standardized SNARC effect slopes were computed by using the individual regression method (unstandardized, Fias et al., 1996) $)^{8}$ and by calculating Pearson correlations between dRTs and digit magnitude, respectively (standardized). Fourth, the standardized regression slopes were Fisher ztransformed to approximate a normal distribution.

For each approach, the next step was then to calculate the $80 \%, 90 \%, 95 \%$, and $99 \% \mathrm{Cls}$ from these data. Because the $90 \% \mathrm{Cl}$ seems to present a relatively good balance between width of the interval and the level of confidence, this $\mathrm{Cl}$ will be discussed in more detail ${ }^{9}$.

Psychometric approach. Reliabilities were calculated using the split-half method by splitting the valid trials into two parts based on order of appearance with the odd-

\footnotetext{
${ }^{7}$ Therefore, the descriptive statistics (e.g., mean RTs and SNARC effect) reported here might differ slightly from values presented in the corresponding papers already published.

${ }^{8}$ See Appendix A for more details regarding the data analysis algorithm used and $\mathrm{R}$ scripts for running the analyses. Note that in case of designs where only two conditions are compared, there are more elaborated methods of estimating reliability (see e.g., Rouder et al., 2019).

${ }^{9}$ Interested readers can conduct the analyses for any other confidence level using the data and analysis scripts shared along with this paper (see below).
} 
even method. For each part and each participant, regression slopes were correlated with each other to obtain a reliability index for the unstandardized and standardized SNARC effect. Then, the Spearman-Brown correction was applied to adjust for (double) task length. Finally, SNARC effect slopes, SDs and reliability estimates were used to determine $80 \%, 90 \%, 95 \%$, and $99 \%$ Cls around the individual unstandardized and standardized slopes by calculating the SEM. This procedure was applied to each dataset separately. For each dataset, the proportion of participants revealing reliable negative slopes (i.e., Cls not containing zero and negative) or reliable positive slopes (i.e., Cls not containing zero and positive) was determined; the remaining proportion had neither a reliable positive slope, nor a reliable negative slope.

H1 Bootstrapping approach. H1 Bootstrap Cls were calculated by custom-built $R$ scripts. ${ }^{10}$ Within each participant $\times$ number $\times$ hand configuration, a sample of $n$ trials was randomly selected from valid trials within this cell. The $n$ parameter was equal to the number of times a given number was repeated in each response-to-hand assignment (the number of observations sampled in each run of bootstrapping procedures should not exceed the number of data points available; e.g., Rousselet et al., 2019) ${ }^{11}$. Based on this set of trials, unstandardized and standardized slopes were calculated. This procedure was repeated 5000 times. To determine the $80 \%, 90 \%$, $95 \%$, and $99 \%$ Cls, the range containing the mid $80 \%, 90 \%, 95 \%$, and $99 \%$, respectively, of these slopes was then calculated.

HO Bootstrapping approach. HO Bootstrap Cls were calculated by custom-built R scripts. Within each participant $\times$ number configuration, two samples of $n$ trials were

\footnotetext{
${ }^{10}$ All R scripts are available at https://osf.io/n7szg/

${ }^{11}$ We acknowledge that in some cells the number of bootstrap samples was higher than the actual number of data points because some trials were excluded during data preprocessing and the bootstrap script does not account for it.
} 
randomly selected from valid trials within this cell. The $n$ parameter was equal to the number of times a given number was repeated in each response-to-hand assignment. These samples were treated as "left hand" and "right hand" responses and were used to calculate unstandardized and standardized slopes. This procedure was repeated 5000 times. Subsequently, $80 \%, 90 \%, 95 \%$, and $99 \%$ Cls were built around 0, based on the range containing respectively the mid $80 \%, 90 \%, 95 \%$, and $99 \%$ of these slopes. Finally, it was checked whether a given participant's SNARC effect slope was outside of that $\mathrm{Cl}^{12}$.

Consistency between psychometric and bootstrapping approaches. To explore the consistency between the three approaches, the proportion of participants who were classified as belonging to the same category for each pair of methods as well as for all three methods together was calculated both for unstandardized and standardized slopes.

Factors influencing the proportion of participants revealing a (reliable) SNARC effect. Using study-level calculations ( $n=18$ data sets), we checked which variables influenced the proportion of participants revealing SNARC slopes smaller than zero as well as the proportion of participants revealing reliable SNARC effects.

Heterogeneity of effects. In order to check whether between-study differences were not solely due to random error, we tested for heterogeneity of effects using the MAJOR package of the JAMOVI software (The jamovi project, 2019) ${ }^{13}$. Specifically, we used the meta-analytic function for testing correlations (for reliability estimates and SNARC

\footnotetext{
12 Importantly, the bootstrapping method used for calculating the $\mathrm{HO} \mathrm{Cl}$ also allows to check how many of the $\mathrm{HO}$ bootstrap slopes were equal to or smaller than the empirical slope for a given participant. This proportion meets the traditional definition of the $p$ value, being the probability of obtaining the difference as empirically observed or larger if the null hypothesis is true. Such a $p$ value can be interpreted as reflecting the generalizability of the effect within a given subject (Onghena, 2020).

13 JAMOVI files are shared at https://osf.io/n7szg/
} 
slopes) and tests for proportions. We report the $R^{2}$ values, which correspond to the percentage of variation between studies due to heterogeneity rather than chance. According to COCHRANE guidelines (Higgins et al., 2020), $I^{2}$ values of $30 \%-60 \%$ correspond to moderate heterogeneity and values of $50 \%-90 \%$ represent substantial heterogeneity ${ }^{14}$. Additionally, $p$ values are reported. Since the null hypothesis of homogeneity is tested, significant effects indicate heterogeneity.

Reality checks. It could potentially be that bootstrap confidence intervals are simply a function of the slope (e.g., smaller slopes are associated with less consistent response pattern and thus larger $\mathrm{Cl}$ ). To check whether bootstrap $\mathrm{Cl}$ brings additional information above and beyond the value of the slope, for each study we correlated the width of the $90 \% \mathrm{H} 1$ bootstrap $\mathrm{Cl}$ with the size of the slope and used meta-analytic techniques to obtain an averaged correlation estimate.

\section{RESULTS}

\section{Overview}

Table 3 summarizes the results of all analyses. The proportion of trials that were retained for SNARC effect calculation was very similar across datasets. In all datasets, robust SNARC effects were observed at the sample level as confirmed with onesample $t$-tests (all $p$ s at least $\leq .047$; in 15 cases $p<.001$ for both unstandardized and standardized slopes). Boxplots illustrating the distribution of slopes across all experiments are presented in Appendix D. Standardized slopes ${ }^{15}$ (before applying Fisher z-transformation) were tested for heterogeneity using meta-analytical

\footnotetext{
${ }^{14}$ COCHRANE guidelines contain this overlap in ranges for the $\mathrm{I}^{2}$, we cite them here as provided in the Handbook.

${ }^{15}$ Calculating heterogeneity requires estimating effect sizes. Because standardized slopes themselves can be considered as effect sizes, running similar tests for unstandardized slopes (which would require transforming them to effect size units) would be redundant in that case.
} 
approaches. The analysis did not reveal any significant differences between studies $(R$ $<.001 \%, p=.903$; see supplementary JAMOVI file).

\section{Reliability}

Datasets differed considerably with regards to the reliability of the SNARC effect. In 10 cases, reliabilities of unstandardized SNARC slopes were satisfactory (i.e., $\geq .65$ ) but mostly lower than typical psychometric standards for individual diagnostic purposes (for personality assessment for adults, diagnostics textbooks often require at least $=$ .80). Heterogeneity analysis indicated systematic variation in reliability estimates between studies $\left(R^{2}=75.88 \% p<.001\right.$ and $R^{2}=71.13 \%, p<.001$ for unstandardized and standardized slopes, respectively).

There were no systematic differences between reliabilities of unstandardized and standardized slopes. In 13 data sets, reliabilities were higher for unstandardized slopes, while, in one case, they were equal for unstandardized and standardized slopes up to the second decimal (Table 3). 
Table 3. Summary of results. The full size and editable table can be accessed at https://osf.io/n7szg/

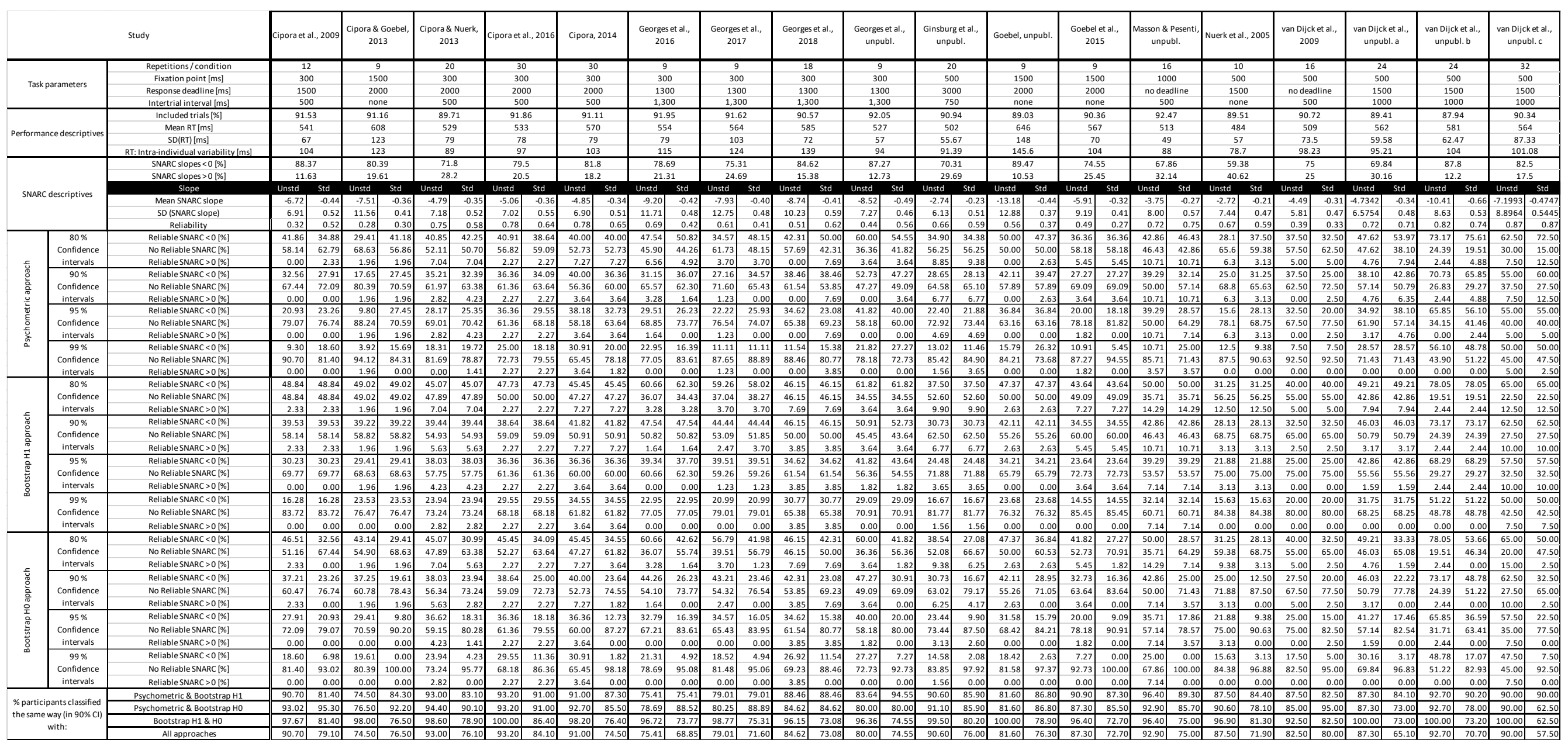




\section{Prevalence of the SNARC effect}

Weighted averages (considering sample sizes in each study) of the proportion of participants revealing a reliable SNARC, a reliable reversed SNARC, or no SNARC effect for different confidence levels for all three methods is summarized in Figure 1; exact percentages for each study are presented in Table 3.

No confidence intervals. Without taking confidence intervals into account, the proportion of participants revealing a SNARC and a reversed SNARC effect is the same for unstandardized and standardized SNARC slopes. The proportion of participants revealing positive and negative slopes differed between studies (from 59\% to $89 \%$ for negative slopes). This range is in line with the proportions reported in the literature (Table 1). Systematic differences were confirmed by heterogeneity analysis $\left(I^{2}=49.86 \%, p=.006\right)$. The weighted average (considering sample size) of participants revealing negative slopes was $76.77 \%$. 
A: Unstandardized slopes

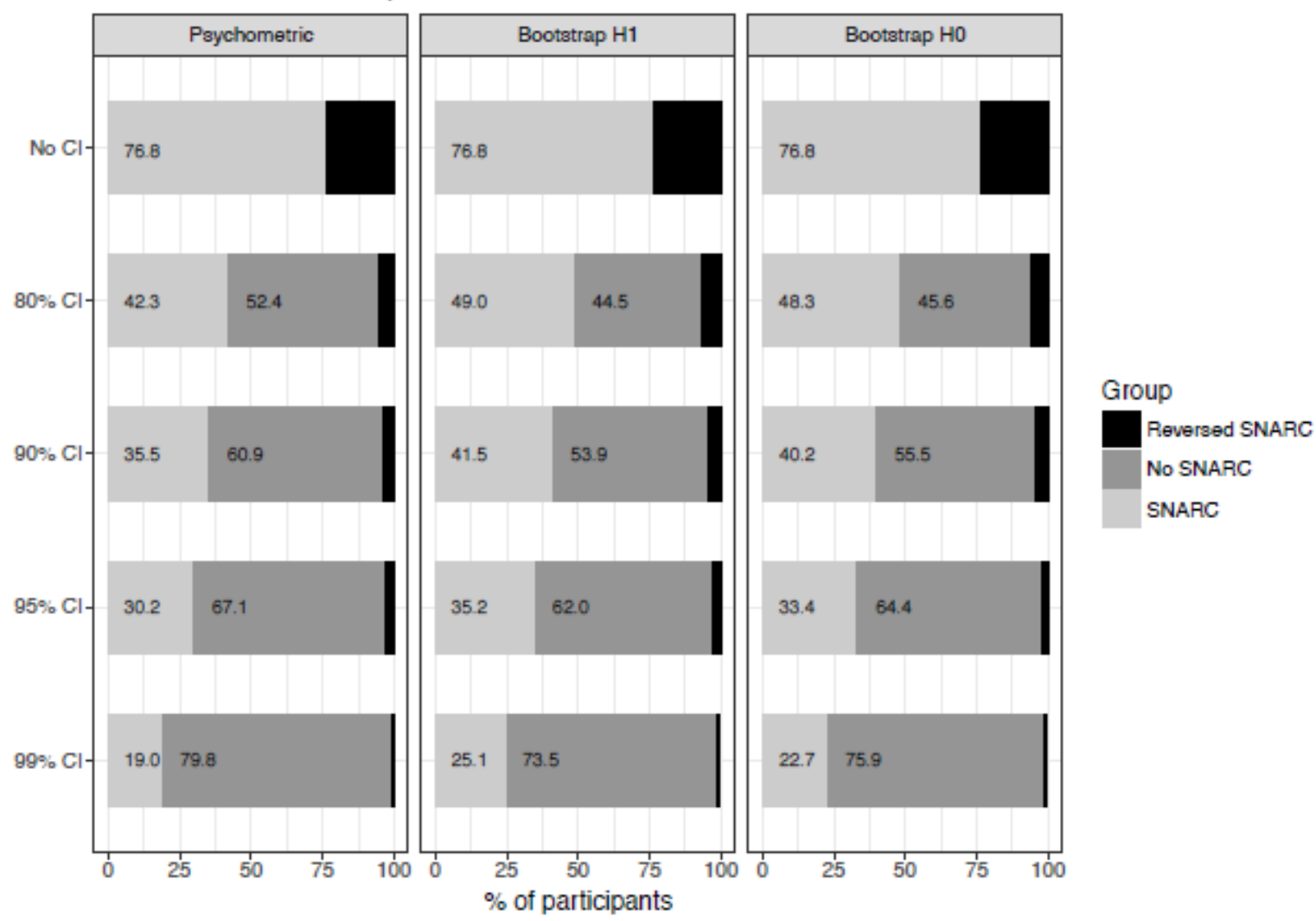

B: Standardized slopes
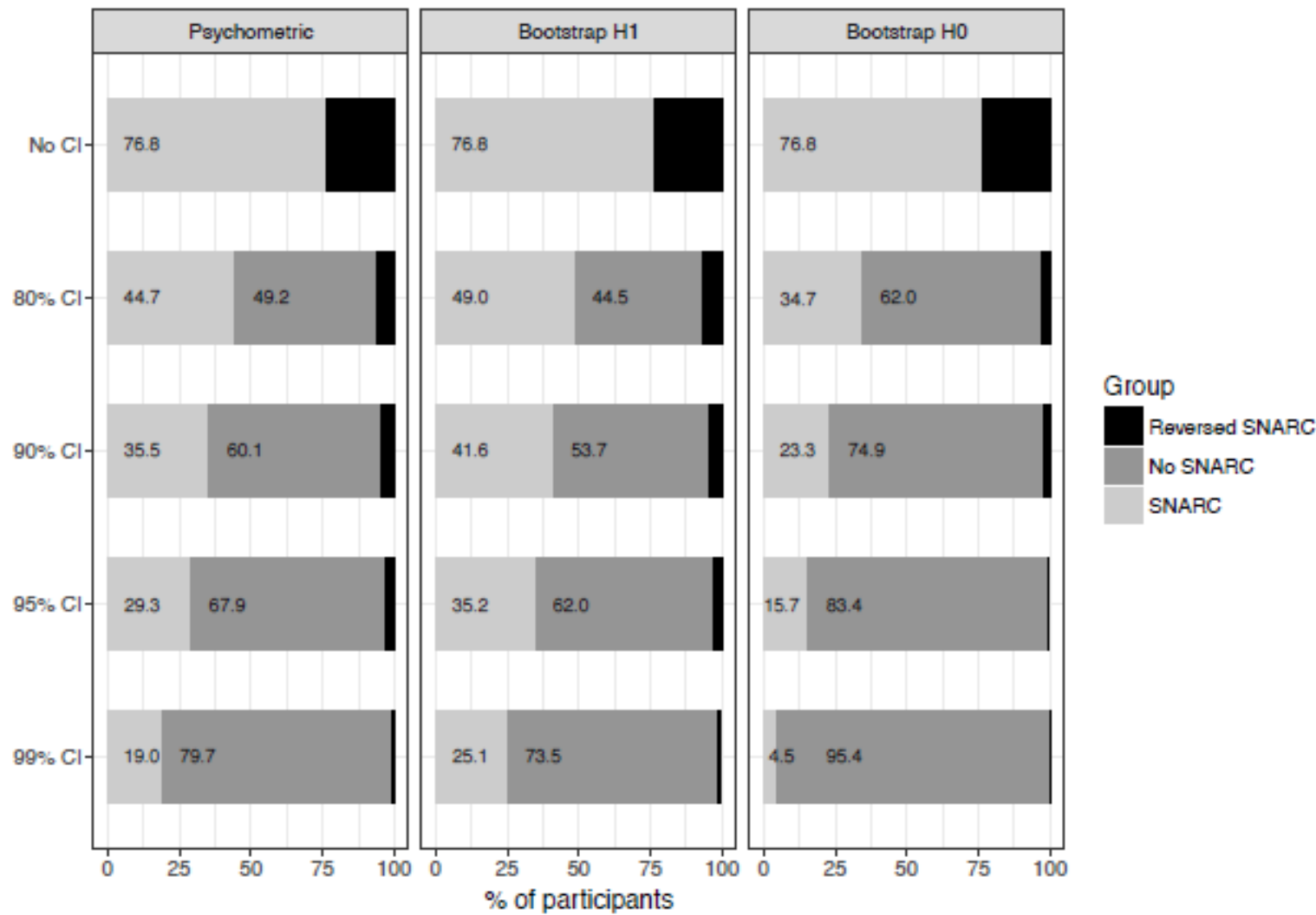

Figure 1. Weighted (by sample size) proportion of participants revealing either a SNARC effect, no SNARC effect, or a reverse SNARC effect for different confidence levels within the psychometric, bootstrapping $\mathrm{H} 1$, and bootstrapping $\mathrm{H} 0$ approaches. Panel A represents results for unstandardized SNARC slopes, and Panel B for standardized ones. Respective 
percentage values are presented inside corresponding parts of bars. The top bar represents the proportion of negative and positive slopes when no confidence intervals are considered. Note that the top bars are duplicated in each column to enable direct comparison with proportions.

Psychometric approach. As expected, the proportion of participants revealing a reliable SNARC, a reliable reversed SNARC, or no SNARC effect varies considerably depending on the confidence level chosen. A more detailed look at the $90 \%$ Cls shows that, across studies, we can say that about $20 \%$ to $70 \%$ (weighted average $35.5 \%$ for both unstandardized and standardized slopes) of participants reveal a reliable SNARC effect. These proportions differed significantly between studies as indicated by heterogeneity analyses $\left(I^{2} \mathrm{~s} \geq 63.46 \%, p s<.001\right)$. There were some minor differences between estimates for unstandardized and standardized slopes, but these were not systematic between studies. Noteworthy, the proportion exceeded $50 \%$ only in the case of three studies and was below $45 \%$ in all other 15 cases. Thus, the prevalence of a reliable SNARC effect (with $90 \%$ confidence interval) is lower than the proportion of negative slopes. On the other hand, the proportion of participants revealing a reliable reverse SNARC effect is very low (never more than $13 \%$ and typically below $5 \%$, weighted average $3.6 \%$ and $4.4 \%$ for unstandardized and standardized slopes, respectively). Interestingly, we found no evidence either for a reliable SNARC or a reliable reverse SNARC effect in $27 \%$ to $80 \%$ of participants.

H1 bootstrapping approach. Again, the proportion of participants revealing a reliable SNARC, a reliable reversed SNARC, or no SNARC effect differed considerably across the chosen confidence levels. A closer look at the $90 \% \mathrm{Cl}$ shows that the proportion of participants revealing reliable negative slopes varied between $30 \%$ and $75 \%$ (with weighted averages of $41.5 \%$ and $41.6 \%$ for unstandardized and standardized slopes, 
respectively). These proportions differed significantly between studies as indicated by heterogeneity analyses $\left(R_{S} \geq 63.59 \%, p s<.001\right)$. Proportions did not systematically differ between unstandardized and standardized slopes. Again, the proportion of individuals revealing a reliable reverse SNARC effect never exceeded $11 \%$ (weighted averages of $4.6 \%$ and $4.7 \%$ for unstandardized and standardized slopes, respectively). Interestingly, we found no evidence for either a reliable SNARC effect or a reliable reverse SNARC effect in more than half of the participants.

HO bootstrapping approach. As with the other methods, the proportion of participants revealing a reliable SNARC, a reliable reversed SNARC, or no SNARC effect differed considerably between confidence levels. A closer look at the $90 \% \mathrm{Cl}$ shows that the proportion of participants revealing reliable negative slopes varied between $20 \%$ and $75 \%$ (weighted average $40.2 \%$ and $23.3 \%$ for unstandardized and standardized slopes, respectively). As indicated by heterogeneity analyses, the proportions differed significantly between studies for the unstandardized slopes $(R=$ $66.84 \%, p<.001)$, but not for the standardized slopes $\left(R^{2}=31.48 \%, p=.072\right)$. The proportion was always lower for standardized slopes. Again, the proportion of a reliable reverse SNARC effect never exceeded $10 \%$ (weighted average $4.3 \%$ and $1.8 \%$ for unstandardized and standardized slopes, respectively). Again, we found no evidence for either reliable a SNARC effect or a reliable reverse SNARC effect in more than half of the participants.

Comparison between the psychometric and bootstrapping approaches

The proportion of participants who were classified as belonging to the same category with all three methods when the $90 \%$ Cls were considered varied between $74.5 \%$ and $93.2 \%$ for unstandardized and $57.5 \%$ and $84.1 \%$ for standardized slopes. 
Congruencies between all pairs of methods were very similar (Table 3). Study-level calculation revealed that the proportion of participants showing a reliable SNARC effect differed significantly between methods both for unstandardized, $F(2,34)=9.70, p<$ $.001, \eta_{p}^{2}=.36$, as well as for standardized SNARC slopes, $F(2,34)=157.98, p<.001$, $\eta_{p}^{2}=.90$. For unstandardized SNARC slopes, the average proportion was smallest for the psychometric method compared to both bootstrapping methods ( $p s \leq .011$, HSD corrected), whereas proportions did not differ between bootstrapping methods ( $p=$ .478 , HSD corrected). For the standardized slopes, proportions differed between all methods ( $p s<.001, \mathrm{HSD}$ corrected), with the lowest average proportion for the H0 bootstrapping method, and the largest one for the $\mathrm{H} 1$ bootstrapping method ${ }^{16}$.

At the study level, correlations between proportions of participants revealing reliable SNARC effects were very high ${ }^{17}$. For unstandardized slopes, proportions estimated with the psychometric approach were highly correlated with the proportions estimated with the $\mathrm{H} 1$ bootstrap $(r=.84, p<.001)$ and with the $\mathrm{H} 0$ bootstrap $(r=.83, p<.001)$. The correlation between proportions estimated with the two bootstrap methods was $r$ $=.99(p<.001)$. For standardized SNARC slopes, the respective correlations were $r=$ $.94(p<.001), r=.86(p<.001)$, and $r=.93(p<.001)$. A similar pattern of results was observed for the reliable reverse SNARC effect (see supplementary JAMOVI files for details).

Factors related to proportions of participants revealing (reliable) SNARC slopes

\footnotetext{
${ }^{16}$ Analyses were conducted on proportions transformed according to the $2 * A R C S I N(S Q R T$ (proportion)) formula. However, analyses on untransformed proportions show virtually the same results.

${ }^{17}$ Note that the heterogeneity analysis shows that studies differed significantly in this respect, which justifies such an analysis.
} 
Because the heterogeneity analysis yielded significant differences between studies regarding proportions of participants revealing (reliable) SNARC slopes, we analyzed which factors were related to these proportions.

No confidence intervals considered. The proportion of participants revealing negative SNARC slopes was related to slope size both for unstandardized and standardized slopes $(r=-.79, p<.001 ; r=-.81, p<.001$ respectively): in data sets showing a more pronounced SNARC effect, the proportion of participants revealing negative slopes was larger. The proportion of participants revealing negative SNARC slopes was also related to RT characteristics, such as mean RT $(r=.65, p=.004)$ and RT variability $(r=.60, p=.008)$ : longer and more variable RTs corresponded to larger proportions of participants revealing negative slopes. On the other hand, this proportion was not related to the SD of the unstandardized $(r=.31, \mathrm{p}=.210)$ and standardized $(r=-.06, p=.824)$ slopes and to slope reliability $(r=-.23, p=.349$ for unstandardized and $r=-.05, p=.839$ for standardized slopes).

Confidence intervals considered. The proportion of individuals revealing negative SNARC slopes was higher in studies in which the mean slope was steeper. With the psychometric approach, it marginally relates to the mean unstandardized slope ( $r=-$ $.40, p=.097)$ and more strongly to the mean standardized slope $(r=-.78, p<.001)$. In the case of $\mathrm{H} 1$ bootstrapping, the correlation with mean slopes is even more pronounced $(r=-.58, p=.012$ and $r=-.88, p<.001$ for unstandardized and standardized slopes, respectively). For $\mathrm{H} 0$ bootstrapping, $r$ equals $-.55(p=.018)$ and $-.91(p<.001)$ for unstandardized and standardized slopes, respectively.

Unsurprisingly, the proportion of participants revealing reliable SNARC effects was higher in data sets in which more participants revealed negative SNARC slopes (psychometric approach: $r=.50, p=.034$ for unstandardized, $r=.44, p=.071$ for standardized slopes; $\mathrm{H} 1$ bootstrapping approach: $r=.55, p=.017$ for unstandardized, 
$r=.56, p=.016$ for standardized slopes; H0 bootstrapping: $r=.52, p=.026$ for unstandardized, $r=.63, p=.005$ for standardized slopes).

The proportion of participants revealing reliable SNARC effects was also related to task reliability. Higher reliability was related to a larger proportion of participants revealing a reliable SNARC effect in all analyses: for psychometric $(r=.44, p=.065$ and $r=.63, p=.005$, for unstandardized and standardized slopes respectively) and bootstrapping approaches (for $\mathrm{H} 1, r=.41, p=.097$ and $r=.51, p=.030$, for unstandardized and standardized slopes, respectively; for $\mathrm{HO}, r=.47, p=.052$ and $r$ $=.43, p=.076$, for unstandardized and standardized slopes, respectively $)^{18}$. However, inspection of the respective scatterplots (see supplementary JAMOVI files) shows that these correlations were mostly driven by two studies (van Dijck et al., unpublished b and c), yielding relatively high reliability estimates compared to the remaining studies.

Furthermore, for the psychometric approach, the proportion of participants revealing reliable negative slopes correlated moderately with the number of repetitions $(r=.47$, $p=.048$ and $r=.44, p=.066$, for unstandardized and standardized slopes, respectively). This was likely due to higher reliabilities obtained in these studies. This relationship did not reach significance with the bootstrapping approaches $(H 1: r=.35$, $p=.150$ and $r=.34, p=.172$, for unstandardized and standardized slopes, respectively; HO: $r=.40, p=.101$ and $r=.32, p=.196$, for unstandardized and standardized slopes, respectively). A larger number of repetitions was thus related to a larger proportion of individuals revealing a reliable SNARC effect. This is likely to be due to the fact that with greater reliability, the Cls (no matter which method was used

\footnotetext{
18 Please note that in several instances correlations do not reach significance and that we do not correct for multiple comparisons here (it is not clear for how many comparisons to correct). Therefore, these results should be interpreted with caution.
} 
to estimate them) get narrower, and more individuals can be classified as revealing a reliable SNARC effect.

Complementary results showed that the proportion of participants revealing a reliable reverse SNARC effect increased with an increasing number of repetitions and reliability (see Supplementary JAMOVI files).

Reality checks: slope and width of H1 bootstrap Cl.

In the last step, we checked whether the width of the bootstrap confidence interval was simply a function of the size of the slope. Thus, in each dataset we looked at correlations between participants' slope and the width of their $\mathrm{H} 1$ bootstrap confidence interval. Because we wanted to inspect the relationship between the precision of the measurement (expressed by the width of the $\mathrm{Cl}$ ) and the size of the SNARC effect irrespective of the direction of the effect, we used the absolute size of the SNARC slope, and used meta-analytic techniques to obtain estimates of these correlations. As a negative slope typically reflects a regular SNARC effect, all absolute values were converted into negative values to facilitate the interpretation of the correlations. The correlations were negative for both unstandardized and standardized slopes $(r=-.313$, $p<.001$ and $r=-.105, p=.005$; see supplementary JAMOVI files), which indicates that a more pronounced SNARC effect was related to a wider $\mathrm{CI}$, but the effect was at best moderate. Results remained unchanged when we considered only negative slopes, or when we did not change the sign of positive slopes (see supplementary JAMOVI files).

\section{DISCUSSION}

\section{Overview}


There are two broadly independent traditions in scientific psychology (Cronbach, 1957): one that focuses on universal group-level phenomena (experimental psychology), and another which aims to understand the nature of individual differences (differential psychology). In the current study, we explored several ways of bridging this gap by large-scale investigations of the individual prevalence of a well-established group-level cognitive phenomenon (the SNARC effect). Using psychometric and newly developed bootstrapping techniques on 18 uniformly preprocessed datasets from different labs, we show that the SNARC effect is only present in a minority of the participants. These results clearly show that significant group-level results cannot be considered as evidence that a cognitive effect is present in the entire population, questioning the universality of the effect and uncovering a potential ecological fallacy (Fisher et al., 2018). In the following section, we first discuss the implications for the SNARC effect before elaborating on the broader theoretical and methodological implications for scientific psychology in general.

\section{Chasing the SNARC effect}

\section{Prevalence of the SNARC effect}

The uniform reanalysis of 18 data sets revealed the presence of a SNARC effect at the sample/group level in each data set, and the pattern of $p$ values at the sample level (15 out of $18 p s<.001)$ indicates its robustness. Please note that we considered all published and unpublished datasets we could access at the time; therefore, we believe that the results should not be influenced by publication bias. We are also not aware of any study which failed to observe the SNARC effect in a typical parity judgment setup in healthy Western adults ${ }^{19}$. The slope estimates as well as the proportion of

\footnotetext{
19 The goal of this study was not to replicate the SNARC effect as such, but to investigate its individual prevalence. Our conclusions actually argue against widely held opinions about the SNARC effect and show that
} 
participants revealing a regular (i.e., smaller than zero) SNARC slope were similar to those previously reported in the literature (Table 1; see also Wood et al., 2008 for a meta-analysis). However, out of the individual participants showing a negative slope, we demonstrate that a reliable non-zero effect at the individual level was present only for a minority, i.e., about $35 \%$ of all participants for the psychometric approach (Figure 1). This proportion was slightly larger with the bootstrapping approaches but did still not exceed $45 \%$ of the participants. Interestingly, a reliable reverse SNARC effect was very rare. Irrespective of the estimation method, it was present in less than $5 \%$ of participants and never exceeded $13 \%$. These proportions refer only to the effect for the data from a single experimental session. Investigating whether the presence of a reliable effect is stable across multiple experimental sessions administered to the same participant can be even more challenging.

Crucially, in about $55 \%$ of the participants, we did not find a reliable SNARC effect. It is important to note that this refers to the absence of evidence for the SNARC effect rather than evidence for no SNARC effect in these participants. There could be many reasons why the effect is not observed in these participants (see below). On the other hand, there are also several points suggesting that, at least in some of these participants, the SNARC effect is truly absent. The first point is that the reliability of the SNARC effect is typically within a reasonable range (see Table 3 ) and not significantly correlated (if anything, only marginally driven by 2 studies) with the proportion of participants showing a reliable SNARC effect. This suggests that the large proportion of subjects who do not show a SNARC effect is not due to an unsuited/unreliable task. Furthermore, the precision of the SNARC measurements in the group that does not

its prevalence is lower than typically assumed. We would like to thank Daniel Lakens, who raised the possible publication bias issue. 
show a reliable SNARC effect is not lower than in the group that does show a reliable SNARC effect because the correlation between the width of the confidence interval (obtained with the $\mathrm{H} 1$ bootstrapping) and size of the SNARC slope reveals that those who have a stronger SNARC effect (independent of the direction) show wider confidence intervals. This latter finding suggests that the large proportion of participants who did not show a reliable SNARC is also not due to a less precise measurement (i.e., extra variability in the data of an individual, which could be expected if the measurement had been done in a non-optimal, environment or circumstances). Taken together, the sufficient reliability and adequate precision of the measured SNARC effects in the group where no reliable SNARC was found suggests that the effect is truly absent in at least a reasonable proportion of the participants. Notably, in two studies where both reliability and precision were the highest (cf. Table 3; see below), the proportion of participants revealing a reliable SNARC effect did not reach $80 \%$, even when the most liberal $80 \% \mathrm{Cl}$ criterion was considered. This can be an indirect argument that the lower-than-expected prevalence of the SNARC effect cannot be solely attributed to a high type II error.

On the other hand, we need to acknowledge that, as shown in Appendix E, the majority of participants not revealing a reliable SNARC effect had slopes $<0$. This might suggest that at least some of these individuals may, in fact, reveal left-to-right mapping, which was not captured by our method. One possible way to further address this issue would be to apply a Bayesian approach, with which one can quantify evidence directly supporting a lack of the SNARC effect in a given participant. However, such methods are not very straightforward and have not been fully developed ${ }^{20}$ so this goes beyond the scope of this work.

\footnotetext{
${ }^{20}$ We would like to thank J. Haaf and T. Faulkenberry for an interesting discussion on that topic.
} 


\section{(Non)paradoxical SNARC effect reliability}

In contrast to the claim of Hedge and colleagues (2018) that reliability of the SNARC effect needs to be low due to small between-subject variance in the effect, we observed high reliabilities (> .80) in two data sets (van Dijck, unpublished b and especially $\mathrm{c}$ ). In both studies, very heterogeneous samples were studied and tasks with more repetitions were used, so both reliability and precision might have been boosted. In study $b, 1^{\text {st }}$ semester students of the bachelor program in applied psychology were tested. Their educational background was more varied than in case of typical university students, including both professional education (e.g., bakery, tourism, social technical education) as well as general secondary education (e.g., math-modern languages, economy-modern languages, STEM, humanities). In study c, participants from heterogeneous groups and of different ages were tested. Most of them were not university students and several participants did not have a higher education degree. This clearly shows that when testing heterogeneous samples, the SNARC effect can be highly reliable. Reliability strongly depends on the inter-individual variability of the property/trait assessed in the sample. In other words, one must keep in mind that the portability assumption (i.e., the assumption that the measurement instrument is a single entity and is characterized by "inherent" reliability) most likely does not hold (Rouder \& Haaf, 2019). Keeping other parameters constant, an increase in sample variance of true scores leads to an increase in reliability (S. R. Cooper et al., 2017). In some cases, low reliability can still account for at least part of the low prevalence of the effect. It can affect prevalence estimates of both psychometric (due to imprecision of the measurement itself and to low sample variance leading to lower reliability estimates) and bootstrapping methods (only due to imprecision of the measurement). The imprecision of measurement refers to low consistency in the RT pattern within 
individuals (in absolute terms, expressed e.g., in milliseconds). Similarly, if there is no consistent pattern within an individual's RTs, slopes estimated with a bootstrap method would also strongly differ between each other. This could be the case due to task characteristics (e.g., too few repetitions, suboptimal trial timings, see below) or testing conditions, for instance. With this in mind, there are three clear recommendations for future correlational studies: (1) in studies testing individual differences in the SNARC effect in particular, the experimenters should ensure that they recruit heterogeneous samples, at least not limiting themselves to student populations, (2) reliability estimates observed in samples drawn from one population cannot be generalized to different populations and must be determined empirically for each population of interest, (3) no matter which samples are tested, one might increase the precision of the SNARC effect measurement (which would lead to relatively narrow bootstrap Cls).

\section{Improving the reliability and precision of the SNARC effect}

To accurately investigate the prevalence of the SNARC effect, irrespective of the method used, future studies need to ensure sufficient reliability and precision of SNARC effect measurement. Below, we list some recommendations on how to reach this goal. These recommendations are likely also valid for other cognitive phenomena.

Sample heterogeneity. As discussed in the section above, testing heterogeneous samples that truly reflect the variance in the population of interest (e.g., when wanting to make inferences about the general population, one should not test only students) would be the first solution. Nevertheless, it must be noted that low reliabilities are observed in some SNARC studies despite the SNARC effect being much more variable than other robust cognitive phenomena. As such, the claim of Hedge et al. (2018) that low reliability of robust phenomena can be attributed to low between-subject variability 
is not adequate in the case of the SNARC effect (which, despite being more variable, is still sometimes not very reliable possibly due to low precision). However, there are several other means by which reliability and precision can be improved.

Task length. Increasing the number of repetitions per condition can be applied for all diagnostic assessment procedures with homogeneous items. The role of the number of trials in experimental psychology tasks has been recently raised as an important factor to be considered to ensure sufficient power both in behavioral and neuroimaging studies (Baker et al., 2020; Brysbaert, 2019; Chen et al., 2022). When more trials are included for averaging, the errors associated with the single measurements tend to cancel each other out more accurately ${ }^{21}$. In the case of the SNARC effect, increasing the number of repetitions of each number per block up to 20 or 30 seems a reasonable solution because the duration of the task does not change dramatically and still can be well below 20 minutes (for simulations, see Cipora \& Wood, 2017). In the presented analyses, reliability is at least at an acceptable level ( $\geq$ $.66)$ in all studies using 20 and more repetitions ${ }^{22}$. Importantly, increasing the number of trials should improve the bootstrap inferences as well. Overall increase in signal-tonoise ratio (precision) will lead to less variable bootstrap slopes and, in consequence, to more narrow Cls (in general, bootstrap techniques are more accurate when more data is available, Rousselet et al., 2019).

Trial timings. Recently, Brigadoi and colleagues (2018) found that increasing the length of intertrial intervals in the parity task can lead to increased reliability of the SNARC effect, perhaps because participants are more alert before each response due

\footnotetext{
${ }^{21}$ However, this holds true only if the error is random. If there are systematic trends in the experiment, due to fatigue or learning, for example, making the experiment longer might not solve this issue. This should be considered each time one wishes to apply this logic to cognitive phenomena.

${ }^{22}$ This estimate is specific for the SNARC effect. The minimum number of recommended repetitions should be estimated specifically for each phenomenon.
} 
to longer waiting time (see e.g., Vallesi et al., 2006). However, evidence for that comes from only one study so far and thus this needs to be explored further. Unfortunately, we could not test these findings in our data sets as durations of intertrial interval in all studies we considered were relatively similar: they varied from $0 \mathrm{~ms}$ to $1300 \mathrm{~ms}$. The increase in reliability observed in Brigadoi et al. (2018) was observed between the condition with intertrial intervals ranging from $1110-1500 \mathrm{~ms}$ and the condition with intertrial intervals between 6000-10000ms.

To sum up, there are numerous means to improve reliability and measurement precision. If one wishes to use the methods described above to investigate other cognitive phenomena, it would be worthwhile to check for optimal task parameters which influence the stability and robustness of the effect in question. As already discussed, reliability is not only crucial when investigating the prevalence of cognitive effects at the individual level, but also when studying correlations with other cognitive phenomena. Because the correlations between the SNARC effect and other cognitive variables are not the main focus of this paper, we do not report them here but do discuss them in more detail in Appendix F.

Implications for numerical cognition: Spatial-numerical associations - universal mechanism or one of many strategies.

In the domain of numerical cognition, it is widely accepted that numbers are mapped onto space. The SNARC effect is one of the hallmark observations that is typically used to support this point (e.g., Cipora et al., 2018; Hubbard et al., 2005). If mapping numbers onto space is universally how people represent and process numerical information, then this spatial mapping should be highly prevalent in the population and should consequently appear reliably in all participants in virtually all numerical tasks. 
The finding that only about $35 \%$ to $45 \%$ of participants show a reliable SNARC effect raises several fundamental questions: for example, is the SNARC effect a good index of this mapping? Or is the link between numbers and space perhaps not as universal as originally proposed? Whereas reasonable reliability is observed in the majority of our reanalyzed samples, additional empirical work is needed to solve the first question. For this purpose, studies using other number-space tasks (e.g., number interval bisection, random number generation) should be (re-)analyzed using the same approach to see whether higher proportions of individuals with reliable effects can be found. Additionally, further insights into the utility of the SNARC effect as an index of the mapping from numbers to space can be obtained by investigating whether the SNARC effect is stable over time within the same individual (i.e., to determine its testretest reliability).

Alternatively, the SNARC effect might be a good proxy for the mapping of numbers to space. In that case, the robust group-level effects are in fact driven by a spatial mapping, which is only reliably present in less than half of the participants. This could imply that this mapping is not universal. In that case, theories of numerical cognition having an oriented spatial representation as the key medium of our capacity to mentally represent numerical magnitude need to be reconsidered; at the population level, a leftto-right spatial mapping might only be one of several different possible ways to represent numerical magnitude. This is not a novel idea: Galton (1880) already emphasized a large variety of forms of human number representations (see also Seron et al., 1992). Moreover, this idea fits nicely with recent ideas suggesting that the SNARC effect is constructed online during task execution (in working memory) as a function of the specific set of stimuli used in the task (e.g., Abrahamse et al., 2017; Fias \& van Dijck, 2016). Importantly, however, this account should be extended to be 
able to account for the high individual variability in the way the task sets are mentally constructed and organized.

Implications for differential cognitive psychology - where to look for correlations?

Having a method to determine the presence of an effect at the individual level opens several new venues for more detailed analyses of (existing) datasets. For example, besides the overall reaction times and standard deviations, the width of an individual $\mathrm{Cl}$ can be considered as the precision with which the effect is measured. As such, this width can be considered as a way to determine whether the measurement of an effect was sufficiently precise or not (in case the width of an individual $\mathrm{Cl}$ is deviant from the average width in the sample) and can thus be used as a tool to identify potentially outlying subjects. This way of data trimming can be very interesting in the context of correlational research. After all, an imprecise measurement (which is no (bivariate) outlier in absolute size) is unlikely to have a positive influence on the correlation under investigation.

In addition to being a tool for data trimming, the method of identifying group differences is also interesting in itself. Apart from comparing groups in terms of the size of an effect, it is also possible to determine whether groups differ in terms of the proportion of individuals showing the effect or not. Consequently, we could distinguish new ways for investigating individual differences in this field. For example, we can try to investigate factors related to whether an individual reveals a reliable effect or not. Second, among individuals who reliably reveal a specific effect, we could then investigate factors related to the strength of such an effect, as there might be qualitative differences between individuals who reveal an effect or not. Evaluating whether effects are reliable at the individual level could also provide insights in populations which do 
not reveal an effect at the group-level. After all, in theory, it is possible that observed null effects are due to two subgroups showing reliable but opposing effects (which cancel each other out when averaging the data; e.g., van Dijck et al., 2020).

\section{Chasing other cognitive phenomena}

Researchers in experimental psychology only rarely question the universality of well-established group-level cognitive phenomena at the individual level. This dates back to Wilhelm Wundt, who treated individual differences as irrelevant (see, Jensen, 2006). At the same time, the starting point for Wundt was physiology, and he was mostly interested in very basic phenomena in sensation and perception, which are indeed more likely to be present in everybody. One may ask whether or not this is a general mistake of cognitive psychology to generalize this assumption to a broader range of more complex, higher order cognitive phenomena. The presence, robustness, and especially replicability of a certain phenomenon are usually considered to be sufficient to infer universal principles of the mind. Nevertheless, even in the history of this specific domain of research, examples suggest that such an approach can be misleading (Fisher et al., 2018). As initially demonstrated by Navon (1977), there is a bias towards global processing of perceptual information. When participants are presented with stimuli such as a letter $\mathrm{H}$ composed of small letters $\mathrm{S}$, they more efficiently recognize the meaning of the big stimuli, rather than the meaning of its components (i.e., they "see the forest before seeing the trees"; Happé \& Frith, 2006). Despite the main effect being robust and highly replicable (see e.g., Hedge et al., 2018), further investigations show that the size (and even presence) of this general bias can differ considerably between individuals. For instance, (1) it strongly correlates with the cognitive style of field dependence (Poirel et al., 2008); (2) it can be related to autism or personality features related to autism spectrum disorder (Van der Hallen et 
al., 2015 for a meta-analysis); (3) it is related to cultural differences (e.g., McKone et al., 2010); (4) some individuals are characterized by local biases (see e.g., Staudinger et al., 2011). This example is not the only one of this kind. Logie et al. (1996) reports similar robust differences in working memory functioning: only $57 \%$ of individuals showed reliable effects on the individual level, while the dominant view was that these effects reflect general properties of the functioning of visual working memory. Siegler (1987), shows similar robust individual differences in numerical cognition: when averaged across trials and participants it seemed that there was only one strategy children used to solve addition problems. When analyzed more thoroughly, it was revealed that the averaging procedure hid multiple strategies being used.

Our analyses present an even more dramatic example - the effect seems to be driven, not by the majority, but rather by a minority of individuals. A similar observation has been reported recently by Grice and colleagues (2020). They analyzed data on how suppressed affective expressions influence perception of neutral faces. Despite a robust group level effect, the ordinal relationship between scores across three conditions present at the group level was only reflected in 11 out of 45 participants (and five participants revealed exactly opposite pattern). In this context, the recent distinction between dominant and indominant psychological phenomena introduced by Rouder and Haaf (2018) might be useful. Phenomena characterized by dominance should be present in all individuals, and we should not expect that there are individuals showing the reverse effect. For instance, one can hardly expect that some individuals reveal a reverse Stroop effect. On the other hand, in phenomena characterized by indominance, the effect observed at the group level is driven by some individuals. Except from resolving power considerations as discussed by Rouder and Haaf (2018), the dominance-indominance distinction has major theoretical implications for scientific 
psychology. We believe that the approach we proposed can help in distinguishing between dominant and indominant phenomena.

In particular, the bootstrapping approach we use seems to be especially promising. It allows researchers to check whether a phenomenon, under scrutiny, is reliably present at an individual level. Importantly, the logic and methodology in this approach can be easily adapted to any cognitive phenomenon for which individual effects are expressed in terms of slopes, such as the Numerical Distance and Numerical Size effects (Hohol et al., 2020), or the ordinal position effect (van Dijck et al., 2020). Importantly, these studies revealed that the proportion of participants revealing reliable effects are not the same across different effects. The prevalence of a reliable numerical distance effect (which is considered to be very robust) was $91 \%$, whereas for the numerical size effect (which is considered to be weaker), the prevalence was only $29 \%$. The prevalence of the ordinal position effect in kindergarteners was even lower (36\% at a confidence level of $80 \%$ ). Similarly, Guida et al. (2020) report a lower prevalence of the ordinal position effect across adult participants, and this was also confirmed in another study (van Dijck et al., 2022). Therefore, one cannot claim that a generic output of our bootstrapping method is, for example, that a reliable effect is present in fewer than $40 \%$ of individuals.

Importantly, the same logic can be also adapted to other cognitive phenomena. On the associated OSF project (https://osf.io/n7szg/) we are sharing scripts that allow for the calculation of $\mathrm{H} 1$ and $\mathrm{HO}$ in tasks where there are only two conditions (e.g., compatible vs. incompatible; word vs. non-word). Such analyses can be particularly powerful if one gathers multiple datasets collected from the same task, preferably in different labs, and conducts similar uniform analyses to increase reliability and robustness of conclusions. 
Very recently, Grice and colleagues (2020) proposed using the proportion of participants revealing the expected pattern of results as a measure of effect size (see also Grice et al., 2015). In our view this is a worthwhile approach, and, at the same time, we would suggest extending this framework by not only looking at whether the general ordinal pattern of means is reflected, but also by examining whether the effects at an individual level are—at least to some extent—unlikely to be solely due to chance by considering confidence intervals. This would be particularly important if there are two conditions only, so one could expect that just due to chance scores in $50 \%$ of participants would be in line with the hypothesis.

\section{CONCLUSION}

To fully understand the nature of human cognition (and mental functioning in general) it is crucial to formulate theories that are not only able to describe group-level phenomena, but are also able to adequately explain individual differences among humans. Understanding this diversity is a natural next step after uncovering the general principles. We think that experimental psychology is reaching the tipping point of being able to do that. With the development of new analytical and methodological techniques, we can pursue such a goal.

The present work shows that a uniform analysis of existing datasets can bring new insights into the nature of well-established cognitive phenomena. This was achieved by applying psychometric and bootstrapping approaches. In this study, we focused on the prevalence of the SNARC effect. More generally, our work emphasizes the idea that robust cognitive effects within the population might not always be observed in every individual. To better understand the processes underlying such effects, one therefore needs to quantify them at the individual level. This can be achieved by 
calculating $\mathrm{Cls}$ around the true score parameter using both psychometric and bootstrapping approaches.

Even in the case of a robust and highly replicable phenomenon at the group level, it is possible that the effect is only reliably present in a minority of individuals. Such an observation leads to critical questions about the nature of the phenomenon and whether, while being reliably present in fewer than $50 \%$ of individuals, it can be considered as evidence for a general principle of (numerical) cognition. In line with these results, to estimate the prevalence of a phenomena, we recommend using the bootstrap techniques to determine how stable the effect is ( $\mathrm{H} 1$ bootstrapping) and how unlikely the observed effect is, given the null hypothesis is true ( $\mathrm{HO}$ bootstrapping).

The method of uniform analysis of multiple existing data sets can answer several questions about the phenomenon being investigated. Furthermore, a similar logic and methodology for investigating the presence of group-level effects at the individual level can be applied to other cognitive phenomena, and can potentially stimulate insights in other domains of cognitive psychology, allowing for a merging of the two branches of psychology, which, in the past few decades, have developed largely in parallel.

\section{CONTEXT}

On a methodological level, this paper demonstrates how psychometric and bootstrapping methods can be used to better understand supposedly well-known cognitive phenomena. It can also serve as an example of how existing data sets can be unified, re-used, and shared in a large-scale collaborative effort aimed at addressing a particular question, which can lead to new insights, especially at the intersection between experimental cognitive and differential psychology. Such an approach is complementary to traditional meta-analyses and multi-lab initiatives and has some commonalities with integrative data analysis. At a content level, our 
approach can be treated as a first step towards broader investigations into the nature of spatial-numerical associations and their role within numerical cognition: do they reflect a universal mechanism by which humans represent numerical information, or are they only one of several possible strategies of representing numbers? This paper is a follow-up on ongoing work by most co-authors aimed at investigating individual differences in the SNARC effect. Originally, the idea of this collaborative effort emerged in discussions of our endeavors in this domain during the European Workshop on Cognitive Neuropsychology (Bressanone, Italy, January 2017). During the development of these ideas, several other co-authors were invited to contribute. Subsequently, the idea was refined during multiple formal and informal conversations of this topic in conferences, talks, and via Twitter.

\section{ACKNOWLEDGEMENTS}

$\mathrm{KC}$ is funded by Research England via the Centre for Mathematical Cognition. MP is a Senior Research Associate at the Fonds National de la Recherche Scientifique (FRSFNRS, Belgium). NM is supported by grant PDR-T.0047.18 and INTER/FNRS/17/11781524/SNAMATH from the Fonds National de la Recherche Scientifique (FRS-FNRS, Belgium) and the Fonds National de la Recherche (FNR, Luxembourg) to MP and CS. The collection of data contributed by SG was supported by a British Academy/Leverhulme Small Research Grant [SG121544]. HCN is a member of LEAD Graduate School \& Research Network [GSC1028], which is funded within the framework of the Excellence Initiative of the German federal and state governments as well as by two German Research Foundation (DFG) projects examining the understanding and replicability of Spatial-Numerical Associations (DFG NU265/8-1 and DFG-FOR 2718 Teilprojekt NU 265/5-1) 
We are grateful to all the colleagues, who commented on earlier versions of this manuscript, in particular: J. Rouder, J. Haaf, T. Faulkenberry, W. Fias, H. LortieForgues, K. Priftis, and M. Zorzi. We would like to thank Morgan Hess for proofreading the manuscript and Wim Gevers for sharing the unpublished data-set collected by Ginsburg et al.

\section{CONFLICT OF INTEREST}

The authors declare that they have no conflicts of interest. 


\section{REFERENCES}

Abrahamse, E. L., van Dijck, J.-P., \& Fias, W. (2017). Grounding Verbal Working Memory: The Case of Serial Order. Current Directions in Psychological Science, 26(5), 429-433. https://doi.org/10.1177/0963721417704404

Baker, D. H., Vilidaite, G., Lygo, F. A., Smith, A. K., Flack, T. R., Gouws, A. D., \& Andrews, T. J. (2020). Power Contours: Optimising Sample Size and Precision in Experimental Psychology and Human Neuroscience. Psychological Methods, 26(3), 295-314. https://doi.org/10.1037/met0000337

Botvinik-Nezer, R., Holzmeister, F., Camerer, C. F., Dreber, A., Huber, J., Johannesson, M., Kirchler, M., Iwanir, R., Mumford, J. A., Adcock, R. A., Avesani, P., Baczkowski, B. M., Bajracharya, A., Bakst, L., Ball, S., Barilari, M., Bault, N., Beaton, D., Beitner, J., ... Schonberg, T. (2020). Variability in the analysis of a single neuroimaging dataset by many teams. Nature, 582(7810), 84-88. https://doi.org/10.1038/s41586-020-2314-9

Brigadoi, S., Basso Moro, S., Falchi, R., Cutini, S., \& Dell'Acqua, R. (2018). On pacing trials while scanning brain hemodynamics: The case of the SNARC effect. Psychonomic Bulletin \& Review, 25, 2267-2273. https://doi.org/10.3758/s13423-017-1418-1

Brown, V. A. (2020). An introduction to linear mixed effects modeling in R. PsyArXiv. https://doi.org/doi.org/10.31234/osf.io/9vghm

Brysbaert, M. (2019). How Many Participants Do We Have to Include in Properly Powered Experiments? A Tutorial of Power Analysis with Reference Tables. Journal of Cognition, 2(1). https://doi.org/10.5334/joc.72

Button, K. S., loannidis, J. P. A., Mokrysz, C., Nosek, B. A., Flint, J., Robinson, E. S. J., \& Munafò, M. R. (2013). Power failure: Why small sample size undermines the reliability of neuroscience. Nature Reviews Neuroscience, 14(5), 365-376. https://doi.org/10.1038/nrn3475

Chambers, C. (2017). The seven deadly sins of psychology: A manifesto for reforming the culture of scientific practice. Princeton University Press.

Chen, G., Pine, D. S., Brotman, M. A., Smith, A. R., Cox, R. W., Taylor, P. A., \& Haller, S. P. (2022). Hyperbolic trade-off: The importance of balancing trial and subject sample sizes in neuroimaging. Neurolmage, 247. https://doi.org/10.1016/j.neuroimage.2021.118786

Cipora, K. (2014). Between task consistency of the SNARC effect. Poster Presented at XXXIInd European Workshop at Cognitive Neuropsychology.

Cipora, K., Czernecka, K., \& Szymura, B. (2009). Temperamental differences in the magnitude of the SNARC effect. Poster Presented at International Society for the Study of Individual Differences.

Cipora, K., \& Göbel, S. M. (2013). Number - Space associations: Just how reliable is the SNARC effect. Poster Presented on XXXIst European Workshop at Cognitive Neuropsychology.

Cipora, K., He, Y., \& Nuerk, H.-C. (2020). The spatial-numerical association of response codes effect and math skills: why related? Annals of the New York Academy of Sciences, 1477, 5-19. https://doi.org/10.1111/nyas.14355

Cipora, K., Hohol, M., Nuerk, H.-C., Willmes, K., Brożek, B., Kucharzyk, B., \& Nęcka, E. (2016). Professional mathematicians differ from controls in their spatialnumerical associations. Psychological Research, 80(4), 710-726. https://doi.org/10.1007/s00426-015-0677-6

Cipora, K., \& Nuerk, H.-C. (2013). Is the SNARC effect related to the level of mathematics? No systematic relationship observed despite more power, more 
repetitions, and more direct assessment of arithmetic skill. Quarterly Journal of Experimental Psychology, 66(10), 1974-1991.

https://doi.org/10.1080/17470218.2013.772215

Cipora, K., Schroeder, P. A., Soltanlou, M., \& Nuerk, H.-C. (2018). More space, better mathematics: Is space a powerful tool or a cornerstone for understanding arithmetic? In K. S. Mix \& M. T. Battista (Eds.), Visualizing Mathematics: The Role of Spatial Reasoning in Mathematical Thought (pp. 77-116). Springer Cham. https://doi.org/10.1007/978-3-319-98767-5_4 77

Cipora, K., Soltanlou, M., Reips, U.-D., \& Nuerk, H.-C. (2019). The SNARC and MARC effects measured online: Large-scale assessment methods in flexible cognitive effects. Behavior Research Methods, 51(4), 1676-1692.

https://doi.org/10.3758/s13428-019-01213-5

Cipora, K., van Dijck, J.-P., Georges, C., Masson, N., Göbel, S. M., Willmes, K., Pesenti, M., Schiltz, C., \& Nuerk, H.-C. (2022). Eighteen trial-level parity judgment datasets $(n=1016)$ from five countries: benefits of sharing unified data of cognitive tasks. PsyArXiv. https://doi.org/10.31234/osf.io/cz96u

Cipora, K., \& Wood, G. (2017). Finding the SNARC instead of hunting it: A 20*20 Monte Carlo investigation. Frontiers in Psychology, 8(JUL). https://doi.org/10.3389/fpsyg.2017.01194

Colling, L. J., Szücs, D., De Marco, D., Cipora, K., Ulrich, R., Nuerk, H.-C., Soltanlou, M., Bryce, D., Chen, S.-C., Schroeder, P. A., Henare, D. T., Chrystall, C. K., Corballis, P. M., Ansari, D., Goffin, C., Sokolowski, H. M., Hancock, P. J. B., Millen, A. E., Langton, S. R. H., ... McShane, B. B. (2020). Registered Replication Report on Fischer, Castel, Dodd, and Pratt (2003). Advances in Methods and Practices in Psychological Science, 3(2), 143-162. https://doi.org/10.1177/2515245920903079

Cooper, H., \& Patall, E. A. (2009). The Relative Benefits of Meta-Analysis Conducted With Individual Participant Data Versus Aggregated Data. Psychological Methods, 14(2), 165-176. https://doi.org/10.1037/a0015565

Cooper, S. R., Gonthier, C., Barch, D. M., \& Braver, T. S. (2017). The Role of Psychometrics in Individual Differences Research in Cognition: A Case Study of the AX-CPT. Frontiers in Psychology, 8, 1482.

https://doi.org/10.3389/fpsyg.2017.01482

Costafreda, S. G. (2009). Pooling fMRI data: Meta-analysis, mega-analysis and multicenter studies. Frontiers in Neuroinformatics, 3(SEP). https://doi.org/10.3389/neuro.11.033.2009

Crocker, L., \& Algina, J. (1986). Introduction to classical and modern test theory. Rinehart amd Winston, Inc.

Crollen, V., \& Noël, M. P. (2015). Spatial and numerical processing in children with high and low visuospatial abilities. Journal of Experimental Child Psychology, 132, 84-98. https://doi.org/10.1016/j.jecp.2014.12.006

Cronbach, L. J. (1957). The two disciplines of scientific psychology. American Psychologist, 12(11), 671-684. https://doi.org/10.1037/h0043943

Curran, P. J., \& Hussong, A. M. (2009). Integrative Data Analysis: The Simultaneous Analysis of Multiple Data Sets. Psychological Methods, 14(2), 81-100. https://doi.org/10.1037/a0015914

de Hevia, M. D., Veggiotti, L., Streri, A., \& Bonn, C. D. (2017). At Birth, Humans Associate "Few" with Left and "Many" with Right. Current Biology, 27(24), 38793884.e2. https://doi.org/10.1016/j.cub.2017.11.024 
Dehaene, S., Bossini, S., \& Giraux, P. (1993). The mental representation of parity and number magnitude. Journal of Experimental Psychology: General, 122(3), 371-396.

Fabbri, M. (2013). Finger Counting Habits and Spatial-Numerical Association in Horizontal and Vertical Orientations. Journal of Cognition and Culture, 13, 95110.

Fattorini, E., Pinto, M., Rotondaro, F., \& Doricchi, F. (2015). Perceiving numbers does not cause automatic shifts of spatial attention. Cortex, 73, 298-316. https://doi.org/10.1016/j.cortex.2015.09.007

Fias, W., Brysbaert, M., Geypens, F., \& D'Ydewalle, G. (1996). The importance of magnitude information in numerical processing: Evidence from the SNARC effect. Mathematical Cognition, 2(1), 95-110.

https://doi.org/10.1080/135467996387552

Fias, W., Lauwereyns, J., \& Lammertyn, J. (2001). Irrelevant digits affect featurebased attention depending on the overlap of neural circuits. Cognitive Brain Research, 12(3), 415-423. https://doi.org/10.1016/S0926-6410(01)00078-7

Fias, W., \& van Dijck, J.-P. (2016). The temporary nature of number-space interactions. Canadian Journal of Experimental Psychology/Revue Canadienne de Psychologie Expérimentale, 70(1), 33-40. https://doi.org/10.1037/cep0000071

Fischer, M. H. (2008). Finger counting habits modulate spatial-numerical associations. Cortex, 44(4), 386-392. https://doi.org/10.1016/j.cortex.2007.08.004

Fischer, M. H., \& Shaki, S. (2014). Spatial associations in numerical cognition-From single digits to arithmetic. Quarterly Journal of Experimental Psychology, 67(8), 1461-1483. https://doi.org/10.1080/17470218.2014.927515

Fisher, A. J., Medaglia, J. D., \& Jeronimus, B. F. (2018). Lack of group-to-individual generalizability is a threat to human subjects research. Proceedings of the National Academy of Sciences of the United States of America, 115(27), E6106E6115. https://doi.org/10.1073/pnas.1711978115

Galton, F. (1880). Visualised numerals. Nature, 21, 252-256.

Georges, C., Hoffmann, D., \& Schiltz, C. (2016). How math anxiety relates to number-space associations. Frontiers in Psychology, 7(SEP), 1-15. https://doi.org/10.3389/fpsyg.2016.01401

Georges, C., Hoffmann, D., \& Schiltz, C. (2017). How and Why Do Number-Space Associations Co-Vary in Implicit and Explicit Magnitude Processing Tasks? Journal of Numerical Cognition, 3(2), 182-211.

Georges, C., Hoffmann, D., \& Schiltz, C. (2018). Implicit and Explicit Number-Space Associations Differentially Relate to Interference Control in Young Adults With ADHD. Frontiers in Psychology, 9, 775. https://doi.org/10.3389/fpsyg.2018.00775

Gevers, W., Santens, S., Dhooge, E., Chen, Q., Van den Bossche, L., Fias, W., \& Verguts, T. (2010). Verbal-Spatial and Visuospatial Coding of Number-Space Interactions. Journal of Experimental Psychology: General, 139(1), 180-190. https://doi.org/10.1037/a0017688

Göbel, S. M., Maier, C. A., \& Shaki, S. (2015). Which numbers do you have in mind? Number generation is influenced by reading direction. Cognitive Processing, 16(S1), 241-244. https://doi.org/10.1007/s10339-015-0715-8

Goodhew, S. C., \& Edwards, M. (2019). Translating experimental paradigms into individual-differences research: Contributions, challenges, and practical 
recommendations. Consciousness and Cognition, 69, 14-25.

https://doi.org/10.1016/J.CONCOG.2019.01.008

Green, S. B., Yang, Y., Alt, M., Brinkley, S., Gray, S., Hogan, T., \& Cowan, N. (2016). Use of internal consistency coefficients for estimating reliability of experimental task scores. Psychonomic Bulletin and Review, 23(3), 750-763. https://doi.org/10.3758/s13423-015-0968-3

Grice, J. W., Craig, D. P. A., \& Abramson, C. I. (2015). A Simple and Transparent Alternative to Repeated Measures ANOVA. SAGE Open, 5(3). https://doi.org/10.1177/2158244015604192

Grice, J. W., Medellin, E., Jones, I., Horvath, S., McDaniel, H., O’lansen, C., \& Baker, M. (2020). Persons as Effect Sizes. Advances in Methods and Practices in Psychological Science. https://doi.org/10.1177/2515245920922982

Guida, A., Mosinski, F., Cipora, K., Mathy, F., \& Noël, Y. (2020). Spatialization in working memory: can individuals reverse the cultural direction of their thoughts? Annals of the New York Academy of Sciences, 1477, 113-125. https://doi.org/10.1111/nyas.14499

Haaf, J. M., Klaassen, F., \& Rouder, J. N. (2018). A Note on Using Systems of Orders to Capture Theoretical Constraint in Psychological Science. https://doi.org/10.31234/osf.io/a4xu9

Haaf, J. M., \& Rouder, J. N. (2017). Developing Constraint in Bayesian Mixed Models. Psychological Methods, 22(4), 779-798. https://doi.org/10.1037/met0000156

Haaf, J. M., \& Rouder, J. N. (2019). Some do and some don't? Accounting for variability of individual difference structures. Psychonomic Bulletin and Review, 26(3), 772-789. https://doi.org/10.3758/s13423-018-1522-x

Happé, F., \& Frith, U. (2006). The Weak Coherence Account: Detail-focused Cognitive Style in Autism Spectrum Disorders. Journal of Autism and Developmental Disorders, 36(1), 5-25. https://doi.org/10.1007/s10803-0050039-0

Hedge, C., Powell, G., \& Sumner, P. (2018). The reliability paradox: Why robust cognitive tasks do not produce reliable individual differences. Behavior Research Methods, 50(3), 1166-1186. https://doi.org/10.3758/s13428-017-0935-1

Higgins, J. P., Thomas, J., Chandler, J., Cumpston, M., Li, T., Page, M. J., \& Welch, V. (Eds.). (2020). Cochrane Handbook for Systematic Reviews of Interventions (Version 6.). The Cochrane Collaboration.

Hoffmann, D., Mussolin, C., Martin, R., \& Schiltz, C. (2014). The impact of mathematical proficiency on the number-space association. PLOS ONE, 9(1). https://doi.org/10.1371/journal.pone.0085048

Hoffmann, D., Pigat, D., \& Schiltz, C. (2014). The impact of inhibition capacities and age on number-space associations. Cognitive Processing, 15(3), 329-342. https://doi.org/10.1007/s10339-014-0601-9

Hohol, M., Willmes, K., Nęcka, E., Brożek, B., Nuerk, H.-C., \& Cipora, K. (2020). Professional mathematicians do not differ from others in the symbolic numerical distance and size effects. Scientific Reports, 10(1), 1-12. https://doi.org/10.1038/s41598-020-68202-z

Hubbard, E. M., Piazza, M., Pinel, P., \& Dehaene, S. (2005). Interactions between number and space in parietal cortex. Nature Reviews Neuroscience, 6(6), 435448. https://doi.org/10.1038/nrn1684

Hussong, A. M., Curran, P. J., \& Bauer, D. J. (2013). Integrative data analysis in clinical psychology research. Annual Review of Clinical Psychology, 9, 61-89. https://doi.org/10.1146/annurev-clinpsy-050212-185522 
Jensen, A. R. (2006). Clocking the Mind. Mental Chronometry and Individual Differences. Elsevier Ltd. https://doi.org/10.1016/B978-0-08-044939-5.X5000-9

Jonas, C. N., Spiller, M. J., Jansari, A., \& Ward, J. (2014). Comparing Implicit and Synaesthetic Number-Space Associations: Visuospatial and Verbal SpatialNumerical Associations of Response Codes. Quarterly Journal of Experimental Psychology, 67(7), 1262-1273. https://doi.org/10.1080/17470218.2013.856928

Klaassen, F. (2020). Combining evidence over multiple individual analyses. In R. van der Schoot \& M. Miočević (Eds.), Small Sample Size Solutons (pp. 126-138). Routledge.

LeBel, E. P. (2015). A New Replication Norm for Psychology. Collabra, 1(1), 1-13. https://doi.org/10.1525/collabra.23

Little, D. R., Nosofsky, R. M., \& Denton, S. E. (2011). Response-Time Tests of Logical-Rule Models of Categorization. Journal of Experimental Psychology: Learning Memory and Cognition, 37(1), 1-27. https://doi.org/10.1037/a0021330

Logie, R. H., Sala, S. Della, Laiacona, M., Chalmers, P., \& Wynn, V. (1996). Group aggregates and individual reliability: The case of verbal short-term memory. Memory and Cognition, 24(3), 305-321. https://doi.org/10.3758/BF03213295

Lorch, R. F., \& Myers, J. L. (1990). Regression analyses of repeated measures data in cognitive research. Journal of Experimental Psychology. Learning, Memory, and Cognition, 16(1), 149-157. https://doi.org/10.1037/0278-7393.16.1.149

Luck, S. J. (2019). Why experimentalists should ignore reliability and focus on precision. https://lucklab.ucdavis.edu/blog/2019/2/19/reliability-and-precision

Lyons, I. M., Nuerk, H.-C., \& Ansari, D. (2015). Rethinking the implications of numerical ratio effects for understanding the development of representational precision and numerical processing across formats. Journal of Experimental Psychology: General, 144(5), 1021-1035. https://doi.org/10.1037/xge0000094

Maloney, E. A., Risko, E. F., Preston, F., Ansari, D., \& Fugelsang, J. (2010). Challenging the reliability and validity of cognitive measures: The case of the numerical distance effect. Acta Psychologica, 134(2), 154-161. https://doi.org/10.1016/j.actpsy.2010.01.006

Martin, S. R., \& Rast, P. (2020). The Reliability Factor: Modeling individual reliability with multiple items from a single assessment. PsyArXiv. https://doi.org/10.31234/osf.io/kr4xq

Maxwell, S. E., Lau, M. Y., \& Howard, G. S. (2015). Is psychology suffering from a replication crisis?: What does "failure to replicate" really mean? American Psychologist, 70(6), 487-498. https://doi.org/10.1037/a0039400

McKone, E., Aimola Davies, A., Fernando, D., Aalders, R., Leung, H., Wickramariyaratne, T., \& Platow, M. J. (2010). Asia has the global advantage: Race and visual attention. Vision Research, 50(16), 1540-1549. https://doi.org/10.1016/J.VISRES.2010.05.010

Mead, A. D. (2005). Reliability: definintions and estimation. In S. Everitt, Brian \& D. C. Howell (Eds.), Encyclopedia of Statistics in Behaviotal Science (pp. 1733-1735). John Wiley \& Sons.

Meteyard, L., \& Davies, R. A. I. (2020). Best practice guidance for linear mixedeffects models in psychological science. Journal of Memory and Language, 112, 104092. https://doi.org/10.1016/j.jml.2020.104092

Navon, D. (1977). Forest before trees: The precedence of global features in visual perception. Cognitive Psychology, 9(3), 353-383. https://doi.org/10.1016/00100285(77)90012-3

Ninaus, M., Moeller, K., Kaufmann, L., Fischer, M. H., Nuerk, H.-C., \& Wood, G. (2017). Cognitive Mechanisms Underlying Directional and Non-directional 
Spatial-Numerical Associations across the Lifespan. Frontiers in Psychology, 8, 1421. https://doi.org/10.3389/fpsyg.2017.01421

Nuerk, H.-C., Wood, G., \& Willmes, K. (2005). The universal SNARC effect: The association between number magnitude and space is amodal. Experimental Psychology, 52(3), 187-194. https://doi.org/10.1027/1618-3169.52.3.187

Onghena, P. (2020). One by one. The design and analysis of replicated randomized single-case experiments. In R. van de Schoot \& M. Miočević (Eds.), Small Sample Size Solutons (pp. 87-101). Routledge. https://doi.org/10.1038/nmat3269

Pashler, H., \& Wagenmakers, E. (2012). Editors' Introduction to the Special Section on Replicability in Psychological Science. Perspectives on Psychological Science, 7(6), 528-530. https://doi.org/10.1177/1745691612465253

Pinhas, M., Shaki, S., \& Fischer, M. H. (2014). Heed the Signs: Operation Signs have Spatial Associations. Quarterly Journal of Experimental Psychology, 67(8), 1527-1540. https://doi.org/10.1080/17470218.2014.892516

Pinhas, M., Tzelgov, J., \& Ganor-Stern, D. (2012). Estimating linear effects in ANOVA designs: The easy way. Behavior Research Methods, 44(3), 788-794. https://doi.org/10.3758/s13428-011-0172-y

Poirel, N., Pineau, A., Jobard, G., \& Mellet, E. (2008). Seeing the Forest Before the Trees Depends on Individual Field-Dependency Characteristics. Experimental Psychology, 55(5), 328-333. https://doi.org/10.1027/1618-3169.55.5.328

Rouder, J. N., \& Haaf, J. M. (2018). Power, Dominance, and Constraint: A Note on the Appeal of Different Design Traditions. Advances in Methods and Practices in Psychological Science, 1(1), 19-26. https://doi.org/10.1177/2515245917745058

Rouder, J. N., \& Haaf, J. M. (2019). A psychometrics of individual differences in experimental tasks. Psychonomic Bulletin and Review, 26(2), 452-467. https://doi.org/10.3758/s13423-018-1558-y

Rouder, J. N., \& Haaf, J. M. (2020). Are There Reliable Qualitative Individual Difference in Cognition? Preprint on Researchgate. https://doi.org/1

Rouder, J. N., Kumar, A., \& Haaf, J. M. (2019). Why most studies of individual differences with inhibition tasks are bound to fail. PsyArxiv Preprints.

Rousselet, G., Pernet, D. C., \& Wilcox, R. R. (2019). A practical introduction to the bootstrap: a versatile method to make inferences by using data-driven simulations. https://doi.org/10.31234/OSF.IO/H8FT7

Rugani, R., Vallortigara, G., Priftis, K., \& Regolin, L. (2015). Number-space mapping in the newborn chick resembles humans' mental number line. Science, 347(6221), 534-536. https://doi.org/10.1126/science.aaa1379

Sauce, B., \& Matzel, L. D. (2013). The causes of variation in learning and behavior: why individual differences matter. Frontiers in Psychology, 4, 395. https://doi.org/10.3389/fpsyg.2013.00395

Schnuerch, M., Nadarevic, L., \& Rouder, J. N. (2020). The truth revisited: Bayesian analysis of individual differences in the truth effect. Psychonomic Bulletin and Review, 1-16. https://doi.org/10.3758/s13423-020-01814-8

Schwarz, W., \& Müller, D. (2006). Spatial associations in number-related tasks: A comparison of manual and pedal responses. Experimental Psychology, 53(1), 415. https://doi.org/10.1027/1618-3169.53.1.4

Seron, X., Pesenti, M., Noël, M.-P., Deloche, G., \& Cornet, J.-A. (1992). Images of numbers, or "when 98 is upper left and 6 sky blue." Cognition, 44(1-2), 159-196. https://doi.org/10.1016/0010-0277(92)90053-K 
Shaki, S., \& Fischer, M. H. (2008). Reading space into numbers - a cross-linguistic comparison of the SNARC effect. Cognition, 108(2), 590-599. https://doi.org/10.1016/j.cognition.2008.04.001

Shaki, S., Fischer, M. H., \& Petrusic, W. M. (2009). Reading habits for both words and numbers contribute to the SNARC effect. Psychonomic Bulletin and Review, 16(2), 328-331. https://doi.org/10.3758/PBR.16.2.328

Shrout, P. E., \& Rodgers, J. L. (2018). Psychology, Science, and Knowledge Construction: Broadening Perspectives from the Replication Crisis. Annual Review of Psychology, 69, 487-510. https://doi.org/10.1146/annurev-psych122216-011845

Siegler, R. S. (1987). The Perils of Averaging Data Over Strategies: An Example From Children's Addition. Journal of Experimental Psychology: General, 116(3), 250-264. https://doi.org/10.1037/0096-3445.116.3.250

Silberzahn, R., Uhlmann, E. L., Martin, D. P., Anselmi, P., Aust, F., Awtrey, E., Bahník, Š., Bai, F., Bannard, C., Bonnier, E., Carlsson, R., Cheung, F., Christensen, G., Clay, R., Craig, M. A., Dalla Rosa, A., Dam, L., Evans, M. H., Flores Cervantes, I., ... Nosek, B. A. (2018). Many Analysts, One Data Set: Making Transparent How Variations in Analytic Choices Affect Results. Advances in Methods and Practices in Psychological Science, 1(3), 337-356. https://doi.org/10.1177/2515245917747646

Simonsohn, U., Simmons, J. P., \& Nelson, L. D. (2020). Specification curve analysis. Nature Human Behaviour, 4(11), 1208-1214. https://doi.org/10.1038/s41562020-0912-z

Staudinger, M. R., Fink, G. R., Mackay, C. E., \& Lux, S. (2011). Gestalt perception and the decline of global precedence in older subjects. Cortex, 47(7), 854-862. https://doi.org/10.1016/J.CORTEX.2010.08.001

The jamovi project. (2019). jamovi (1.0).

Thiele, J. E., Haaf, J. M., \& Rouder, J. N. (2017). Is there variation across individuals in processing? Bayesian analysis for systems factorial technology. Journal of Mathematical Psychology, 81, 40-54. https://doi.org/10.1016/j.jmp.2017.09.002

Thompson-Schill, S. L., Braver, T. S., \& Jonides, J. (2005). INDIVIDUAL DIFFERENCES: Editorial. Cognitive, Affective, \& Behavioral Neuroscience, 5(2), 115-116. https://doi.org/10.3758/CABN.5.2.115

Toomarian, E. Y., \& Hubbard, E. M. (2018). On the genesis of spatial-numerical associations: Evolutionary and cultural factors co-construct the mental number line. Neuroscience \& Biobehavioral Reviews, 90, 184-199. https://doi.org/10.1016/J.NEUBIOREV.2018.04.010

Tzelgov, J., Zohar-Shai, B., \& Nuerk, H.-C. (2013). On defining quantifying and measuring the SNARC effect. Frontiers in Psychology, 4(MAY), 3-5. https://doi.org/10.3389/fpsyg.2013.00302

Vallesi, A., Shallice, T., \& Walsh, V. (2006). Role of the Prefrontal Cortex in the Foreperiod Effect: TMS Evidence for Dual Mechanisms in Temporal Preparation. Cerebral Cortex, 17(2), 466-474. https://doi.org/10.1093/cercor/bhj163

Van der Hallen, R., Evers, K., Brewaeys, K., Van den Noortgate, W., \& Wagemans, J. (2015). Global processing takes time: A meta-analysis on local-global visual processing in ASD. Psychological Bulletin, 141(3), 549-573. https://doi.org/10.1037/bul0000004

van Dijck, J.-P., Abrahamse, E., \& Fias, W. (2020). Do preliterate children spontaneously employ spatial coding for serial order in working memory? Annals of the New York Academy of Sciences, 1447, 91-99.

https://doi.org/10.1111/nyas.14430 
van Dijck, J.-P., \& Fias, W. (2011). A working memory account for spatial-numerical associations. Cognition, 119(1), 114-119. https://doi.org/10.1016/j.cognition.2010.12.013

van Dijck, J.-P., Fias, W., \& Cipora, K. (2022). Spatialization in working memory and its relation to math anxiety. Annals of the New York Academy of Sciences, 1512(1), 192-202. https://doi.org/10.1111/nyas.14765

van Dijck, J.-P., Gevers, W., \& Fias, W. (2009). Numbers are associated with different types of spatial information depending on the task. Cognition, 113(2), 248-253. https://doi.org/10.1016/j.cognition.2009.08.005

Viarouge, A., Hubbard, E. M., \& McCandliss, B. D. (2014). The cognitive mechanisms of the SNARC effect: An individual differences approach. PLOS ONE, 9(4). https://doi.org/10.1371/journal.pone.0095756

Vogel, E. K., \& Awh, E. (2008). How to exploit diversity for scientific gain. Current Directions in Psychological Science, 17(2), 171-176. https://doi.org/10.1111/j.1467-8721.2008.00569.x

Weston, S. J., Graham, E. K., \& Piccinin, A. M. (2020). Coordinated Data Analysis: A New Method for the Study of Personality and Health. In P. Hill \& A. Mathias (Eds.), Personality and Healthy Aging in Adulthood (pp. 75-92). Springer Nature.

Willmes, K. (2010). The methodological and statistical foundations of neuropsychological assessment. In J. Gurd, U. Kischka, \& J. Marschall (Eds.), The Handbook of Clinical Neuropsychology (Second ed., pp. 28-49). Oxford University Press.

Wood, G., Nuerk, H.-C., \& Willmes, K. (2006a). Crossed hands and the SNARC effect: A failure to replicate Dehaene, Bossini and Giraux (1993). Cortex, 42(8), 1069-1079. https://doi.org/10.1016/S0010-9452(08)70219-3

Wood, G., Nuerk, H.-C., \& Willmes, K. (2006b). Variability of the SNARC effect: Systematic interindividual differences or just random error? Cortex, 42(8), 11191123. https://doi.org/10.1016/S0010-9452(08)70223-5

Wood, G., Willmes, K., Nuerk, H.-C., \& Fischer, M. H. (2008). On the cognitive link between space and number: a meta-analysis of the SNARC effect. Psychology Science Quarterly, 4(4), 489-525. https://doi.org/10.1027/1618-3169.52.3.187

Yang, T., Chen, C., Zhou, X., Xu, J., Dong, Q., \& Chen, C. (2014). Development of spatial representation of numbers: A study of the SNARC effect in Chinese children. Journal of Experimental Child Psychology, 117, 1-11.

https://doi.org/10.1016/J.JECP.2013.08.011 